\title{
The neurocognition of developmental disorders of language
}

Michael T. Ullman ${ }^{1 *}$, F. Sayako Earle ${ }^{2}$, Matthew Walenski ${ }^{3}$, and Karolina Janacsek ${ }^{4,5}$

1. Brain and Language Lab, Department of Neuroscience, Georgetown University, Washington DC, 20057

2. Department of Communication Sciences \& Disorders, University of Delaware, Newark, DE, USA

3. Department of Communication Sciences and Disorders, Northwestern University

4. Institute of Psychology, Eotvos Lorand University, Damjanich utca 41-43, H-1071 Budapest, Hungary

5. Brain, Memory and Language Lab, Institute of Cognitive Neuroscience and Psychology, Research Centre for Natural Sciences, Hungarian Academy of Sciences, Magyar Tudósok Körútja 2, H-1117 Budapest, Hungary

*Correspondence concerning this article should be addressed to Michael T. Ullman. Address: Brain and Language Laboratory; Department of Neuroscience; Georgetown University; Box 571464; Washington, DC 20057-1464. Email: michael@ georgetown.edu. Telephone: (202) 687-6064. Fax: (202) 687-6914. 


\begin{abstract}
Developmental disorders of language include developmental language disorder, motor-speech disorders such as articulation disorder and stuttering, and dyslexia. These disorders have been explained by various accounts, which generally focus on their behavioral rather than neural characteristics, their processing rather than learning impairments, and each disorder separately rather than together, despite their commonalities and comorbidities. Here we update and review a unifying neurocognitive account, the Procedural circuit Deficit Hypothesis (PDH). The PDH posits that abnormalities of brain structures underlying procedural memory (learning and memory that relies on the basal ganglia and associated circuitry) can explain numerous brain and behavioral characteristics, across learning and processing, in multiple disorders, including both commonalities and differences. We describe procedural memory, examine its role in multiple aspects of language, and then present the PDH and relevant evidence across language-related disorders. The PDH has substantial explanatory power, and both basic research and translational implications.
\end{abstract}

Keywords: developmental language disorder (DLD), motor-speech disorders (articulation disorder, childhood apraxia of speech, stuttering), dyslexia, procedural memory, language, Procedural circuit Deficit Hypothesis (PDH)

Acknowledgements: We thank Gregory Ashby, Christopher Conway, Laurie Cutting, Jarrad Lum, Maximillian Riesenhuber, Lauren Russell, Carol Seger, Andrew Tiu, and Christine Weber for helpful input on this paper. This work was supported in part by National Science Foundation BCS 1439290, National Institute of Health R21 HD 087088, and a research grant from the Tourette Association of America, to M.T.U.; by National Institute of Health R21 DC 016391 to F.S.E; and by Hungarian Scientific Research Fund OTKA PD 124148, National Brain Research Program project 2017-1.2.1-NKP-201700002, and a Janos Bolyai Research Fellowship of the Hungarian Academy of Sciences, to K.J. 


\section{INTRODUCTION}

Developmental disorders can impact various aspects of language. Developmental Language Disorder (DLD; superseding 'specific language impairment') broadly refers to childhood language problems not explained by factors such as hearing deficits or environmental deprivation (APA 2013; Bishop et al. 2017). Developmental dyslexia, by contrast, involves reading impairments (Goswami 2015), while motor-speech disorders, such as articulation disorder, childhood apraxia of speech (verbal dyspraxia), and developmental stuttering, affect the production of fluent speech (Alm 2004; Bernthal et al. 2009). These disorders are not uncommon (e.g., 7\% prevalence for DLD) and can persist into adulthood, with important social and economic consequences (Leonard 2014; Elbro et al. 2011).

Various accounts have been proposed to explain these disorders. DLD has generally been explained either by a processing deficit (capacity limitations on processing, or problems with working memory, phonological processing, or temporal processing) or a specific linguistic deficit, especially of grammar, which tends to be strongly affected in DLD (Leonard 2014; Ullman \& Pierpont 2005). Dyslexia has often been accounted for by deficits related to phonology or magnocellular circuitry (Ramus et al. 2003). Motor-speech disorders have generally been explained by impairments in the motor production of speech, with neural accounts for stuttering (Alm 2004; Bernthal et al. 2009).

Though these accounts have some explanatory power (Bernthal et al. 2009; Leonard 2014; Ramus

et al. 2003), important gaps remain. First, many of the accounts are essentially functional, in that they ascribe the problem to a particular function (e.g., working memory). Yet these disorders must ultimately be explained by neurobiological disruptions. Thus, neurocognitive accounts that unify neurobiological and cognitive aspects should have more explanatory power than purely functional (or purely neurobiological) accounts. Second, since these are developmental disorders, impairments in learning may play important roles, perhaps in addition to processing deficits - yet most of the accounts listed above focus on processing rather than learning. Third, most accounts focus on a particular disorder, yet many of the disorders' symptoms overlap, and the disorders are often comorbid with one another (Leonard 2014; Bernthal et al. 2009). Thus, accounts that unify these disorders may be better positioned to advance the field. 
The Procedural Deficit Hypothesis (PDH) attempts to address these gaps (Ullman, 2004; Ullman and Pierpont, 2005). The PDH was originally presented in depth for DLD (Ullman \& Pierpont 2005). According to the PDH, DLD may be largely explained by abnormalities of brain structures underlying procedural memory, which is defined as the learning and memory that relies on the basal ganglia (BG) and its associated circuitry (although the cerebellum may interact with this circuitry we do not focus on the cerebellum here; see Bostan \& Strick 2018; Ullman \& Pierpont 2005). Reflecting the anatomical basis of the PDH, we henceforth refer to it as the Procedural circuit Deficit Hypothesis. The PDH of DLD was motivated by the grammar impairments in DLD, since grammar has been linked to procedural memory as part of the declarative/procedural model of language (Ullman 2004, 2016). According to the PDH, individuals with procedural circuit abnormalities should show deficits not only of grammar and procedural memory, but also of apparently non-procedural functions such as working memory that also depend on this circuitry. However, the PDH posits that declarative memory, defined as the learning and memory that relies on the medial temporal lobe (MTL) and its associated circuitry, remains largely normal in the disorder, and can compensate for procedural circuit deficits. Thus, although the PDH was motivated by functional problems (of grammar), it is a unifying neurocognitive account in that it posits neuroanatomical abnormalities that can explain patterns of spared and impaired learning and processing, across both language and non-language domains.

It is also a unifying account in that it can explain multiple disorders. Although the PDH has been laid out in the greatest depth for DLD, it has also been proposed for dyslexia (Ullman 2004). Nicolson and Fawcett (2007) posited a related account for dyslexia, though they specifically implicate cerebellar circuits. The PDH has not previously been applied to motor-speech disorders, except in the case of the KE family, who are commonly diagnosed with verbal dyspraxia (Ullman \& Pierpont 2005).

Here we provide an up-to-date exposition of the PDH, unifying neural and functional characteristics, for both learning and processing, across DLD, motor-speech disorders, and dyslexia. First, we describe procedural memory with greater specificity than before, integrating findings from several fields. Building on this understanding of procedural memory, we then examine the predicted roles for this system in typical development across multiple aspects of language learning and processing. These prior sections provide the foundation for the PDH and its predictions, which we examine across different developmental disorders. Finally, we conclude with a brief discussion of implications and avenues for future investigation. 


\section{PROCEDURAL MEMORY: BASAL GANGLIA-BASED LEARNING}

\section{What are the basal ganglia?}

The BG in humans and other primates include the striatum (nucleus accumbens, caudate nucleus, and putamen), the globus pallidus (external and internal segments), the subthalamic nucleus, and both the substantia nigra (pars compacta and pars reticulata) and ventral tegmental area in the midbrain (Hélie et al 2015). The BG receive inputs from much of cortex (which mainly project to the striatum), and provide outputs to frontal and other cortical regions, via the thalamus, as well as to subcortical structures. Within the BG, the circuitry is organized into 'direct' and 'indirect' pathways, which together (dis)inhibit frontal and other cortical activity, thereby underlying the selection of motor and other cortically-based representations (Frank 2005). Dopamine plays a key role, with the midbrain structures projecting dopaminergic neurons to the striatum.

The circuitry running through the BG is organized into parallel circuits (partially topographically and functionally segregated 'loops'), each of which receives somewhat different cortical inputs and projects to largely distinct frontal and other (e.g., temporal) cortical regions, which in turn feed back into their respective circuits (Middleton \& Strick 1996). For example, somatosensory-(posterior) putamenfrontal motor circuitry underlies motor functions; posterior parietal-anterior dorsal striatum (anterior caudate/putamen)-prefrontal circuitry subserves cognition, such as working memory and executive functions; while hippocampal/amygdala-ventral striatum (nucleus accumbens and ventral caudate/putamen)-orbitofrontal/anterior cingulate circuitry supports motivational functions (Draganski et al 2008; Seger et al 2010).

\section{How do the basal ganglia learn?}

BG-based learning involves generating predictions about associations, and then evaluating these predictions based on the 'outcome' (information about the correctness of the prediction) (Balleine \& O'doherty 2010). In particular, learning in the BG occurs when such (dis)confirmatory information is promptly available after the prediction is generated. For example, the BG are involved when a rat learns to predict that when it sees a lever (stimulus) it should press it (response), with the goal of receiving an 
immediately subsequent food reward (signaling the prediction was correct). Similarly, in categorization tasks such as the 'weather prediction' task, in which human participants select a (probabilistically) predictable 'output' stimulus (e.g., 'rain') based on an 'input' stimulus (particular cards), rapid (dis)confirmation (feedback) of the predicted association leads to BG-based learning, whereas slow feedback, or none at all, leads instead to learning in the MTL (Foerde \& Shohamy 2011a, Poldrack et al 2001). The knowledge learned in the BG appears to be implicit (not available to conscious awareness) (Janacsek \& Nemeth 2012; Ashby et al. 2007).

Both the input and output elements of the association learned in the BG can be either a stimulus or a response. Thus, one can learn not only stimulus-response and stimulus-stimulus pairs, as in the examples above, but also response-response pairs (e.g., after sneezing one should apologize) and responsestimulus pairs (e.g., if I smile you will smile) (Balleine \& O'doherty 2010, Foerde \& Shohamy 2011b). In both animal and human experimental paradigms the feedback is generally separate from the association (as above), but it does not need to be: when predicting that a given stimulus or response should lead to a subsequent stimulus, the mere occurrence of the latter can serve as feedback (e.g., the next item in a sequence). More generally, the basic selection function of the BG (see above) may be thought of as selecting the predicted output, with learning occurring when information indicates that this selection was incorrect (Frank 2005).

Indeed, BG-based learning ensues from generating incorrect predictions (if a prediction is correct, no learning is needed). Unpredicted outcomes (e.g., the rat failed to predict that pressing the lever will lead to a food reward) create/strengthen the input-output associations that should have been predicted, while incorrect predictions weaken them, respectively via increases or decreases of dopamine in the striatum (Frank 2005). Crucially, such learning occurs if the prediction is incorrect not only about the outcome itself but also when it will occur (e.g., if we incorrectly predict the next item of a sequence will occur immediately). Thus, the BG learn not only to predict what should occur, but also when it occurs.

Because prediction errors are larger earlier during learning, more learning occurs earlier. Additionally, the more predictable the output given the input, the faster the learning. Thus, more predictable (higher probability) pairs are typically learned faster than less predictable pairs, with completely deterministic pairs learned the fastest (Delgado et al 2005). BG-based learning is therefore gradual, but varies (in part) according to the predictability (probability) of the association. Eventually, 
after extensive training, the learned associations become not only very fast and accurate, but also inflexible, that is, less sensitive to prediction errors from the feedback-characteristics of habitual/automatic behaviors (Ashby \& Crossley 2012, Graybiel \& Grafton 2015).

The functional neuroanatomy of BG-based learning may reflect distinctions between earlier and later phases of learning. Anterior portions of the striatum (ventral striatum and anterior caudate/putamen) seem to be more important for earlier phases, while posterior portions (posterior caudate/putamen) play a larger role in later phases. The reasons for this anterior/posterior striatal distinction for earlier/later learning remain unclear, but may be related to the different parallel circuits. Anterior striatal circuits may support aspects of motivation (linked to ventral striatal circuitry; see above) as well as working memory and executive functions (linked to anterior caudate/putamen), which might underlie early-stage predictionfeedback learning of associations; in contrast, posterior portions may underlie aspects of motor and/or visual learning (motor and visual circuits rely on more posterior putamen/caudate) that may take place during the fine-tuning of performance in later stages of acquisition, or perhaps even in the processing of automatized associations (Doyon et al 2009, Seger et al 2010).

Learning in the BG also critically involves cortex. In particular, BG-based learning underlies the creation/strengthening of cortico-cortical connections between cortical neuronal populations that project to the BG (striatum) and those the BG project to (via the thalamus), while these input and output neuronal populations (representing the input and output elements of the association) are both active (Hélie et al 2015). This simultaneous activation allows the formation/strengthening of the cortico-cortical connections via Hebbian learning (they fire together and thus wire together). An increased reliance on these corticocortical connections ('systems consolidation') is associated with increased automatization, though the analogous input-output circuitry passing through the BG may continue to be relied upon (Ashby \& Crossley 2012, Doyon et al 2009). Systems consolidation may promote generalization, for example, to new members of a category in category learning (see below). Consistent with BG inputs originating importantly in parietal regions, and the BG projecting importantly to frontal regions, the cortico-cortical circuits formed by the BG may play an important role in 'dorsal stream' pathways (Ullman 2004, 2016).

\section{What do the basal ganglia learn?}


The associations learned by the BG provide the building blocks for learning various simple and complex functions and behaviors, including habits, skills, sequences, and categories (Ashby \& Crossley 2012, Graybiel \& Grafton 2015). (Their learning can also rely on declarative memory; see below.) Here we focus on the latter three.

A skill can be defined as a serial combination of (perceptuo-) motor and/or cognitive computations, which together constitute a new capacity, and with sufficient practice can become automatized (e.g., driving a car, playing an instrument) (Graybiel \& Grafton 2015). Skill learning is often modelled with perceptuo-motor sequence learning. In humans, this is generally tested with the implicit serial reaction time (SRT) task, in which participants respond to a sequence of stimuli with corresponding buttons, without being informed that a sequence exists (and generally with little or no explicit knowledge of the sequence after learning). Each stimulus typically occurs rapidly after the previous stimulus or response. The sequence in this task can be deterministic and/or probabilistic, in that any given item(s) may be always followed by the same item(s) (deterministic) or by different item(s) with varying probabilities (probabilistic) (Janacsek \& Nemeth 2012). Learning involves predicting a subsequent stimulus, which also serves as feedback; as learning proceeds, anticipation responses become more common (Vakil et al 2017). Associations (dependencies) of adjacent elements seem to be learned faster than of non-adjacent (long-distance) elements, which in turn are learned faster if the dependency (number of non-predictive items between the elements) is smaller (Remillard 2008). Moreover, a dependency that requires more preceding (predicting) elements is learned more slowly than one that requires fewer (Remillard 2008). Converging evidence (e.g., from lesion and neuroimaging studies) suggests the following functional neuroanatomy for perceptuo-motor sequence learning in the implicit SRT and related tasks (Doyon et al 2009, Penhune \& Steele 2012). Early stages of learning depend importantly on the anterior striatum, mainly the anterior caudate/putamen (but not the cerebellum, at least in implicit SRT; Hardwick et al. 2013). In contrast, in later stages, during and after automatization, posterior portions of the caudate and putamen are engaged, as well as cortical (mainly (pre)motor, as well as parietal) regions, though again, apparently not the cerebellum.

Sequence learning is not limited to perceptuo-motor sequences. We also encounter purely perceptual (e.g., auditory, visual) sequences, with no overt responses involved, such as when listening to music. Such sequences are often investigated in 'statistical learning' paradigms that involve the sequential presentation of stimuli (e.g., tones, shapes) without responses, with knowledge typically tested in 
judgment tasks after learning (Durrant et al 2012, Turk-Browne et al 2009). As with perceptuo-motor sequences, the items in these sequences are normally presented predictably and rapidly, and the sequences can be deterministic and/or probabilistic. (Here we only discuss tasks with non-linguistic stimuli; for those with language-related stimuli, including artificial grammars, see below.) These paradigms appear to rely importantly on BG-based learning. Learning, which occurs gradually, appears to involve prediction (e.g., of the subsequent item), resulting in the acquisition of stimulus-stimulus associations; moreover, the learning and knowledge of the sequences are generally implicit (Abla et al 2008, Turk-Browne et al 2009). Functional imaging studies, which have focused on early learning stages, have implicated the anterior caudate (head/body) as well as frontal (mainly premotor) and other cortical regions, though apparently not the cerebellum (e.g., Turk-Browne et al 2009). Literatures other than statistical learning also link the learning or processing of perceptual sequences to the BG. For example, evidence suggests that beat (rhythm) perception ('temporal processing') and aspects of music processing rely on the BG, particularly the striatum (as well as premotor cortex and the cerebellum for music processing) (Grahn \& Rowe 2009).

Category learning involves learning to group elements into categories. It is modelled with various paradigms, though most involve learning to categorize perceptual (usually visual) stimuli (Seger \& Miller 2010). For example, in the weather prediction task, participants learn which visually-presented cards are probabilistically associated with which visually-presented weather outcome (rain or sun). The rules of categorization in such tasks can be deterministic or probabilistic. Category learning typically exhibits characteristics of BG-based learning. Learning is gradual, and is generally implicit in that the categorization rules are not told to the participants, who moreover do not usually acquire this knowledge explicitly (at least for more complex rules) (Seger \& Miller 2010). BG-based category learning involves predicting the category (rain) given the input (the cards), with rapid feedback regarding the correctness of the prediction (Foerde \& Shohamy 2011b). During learning, input element-category associations are acquired and then generalized according to the relevant features (Seger \& Miller 2010). Generalization, that is, the ability to successfully categorize new elements or combinations of elements, appears to take place gradually, and may depend on systems consolidation, in particular the formation of feature-based cortico-cortical connections (between input element and/or category neuronal populations) (Ashby et al. 2007). After substantial practice, categorization measures (selecting the appropriate category based on the input) become automatized: speed and accuracy reach asymptote, secondary tasks do not impair performance, and the categorization behavior becomes inflexible (difficult to modify) (Ashby \& Crossley 
2012, Hélie et al 2010b). Converging evidence suggests that early phases of category learning depend especially on the caudate head and the nucleus accumbens (and in some studies the caudate body and tail), while later phases mainly involve the (posterior) putamen; the cerebellum does not seem to be heavily involved (Seger \& Miller 2010, Wilkinson et al 2014). Cortex is also involved, in particular frontal regions: prefrontal cortex (especially ventral lateral prefrontal cortex) during earlier phases and premotor cortex during later phases and after automatization (Hélie et al 2010a, Waldschmidt \& Ashby 2011).

\section{Basal ganglia- vs. MTL-based learning}

Skills and knowledge that can be learned in the BG (i.e., in procedural memory) may (also) be learned in the MTL (i.e., in declarative memory). Here we summarize MTL learning, and then discuss the factors that modulate BG- vs. MTL-based learning. (We do not discuss other types of learning here, such as purely cortical learning; Seger \& Miller 2010.)

As mentioned above, we define declarative memory as MTL-based learning and memory (Davachi 2006, Eichenbaum 2012, Ullman 2004, 2016). Learning ('encoding') initially relies on the hippocampus and other MTL structures, which link (neo)cortical representations via Hebbian learning. Unlike BG-based learning, MTL-based learning (e.g., observational learning) does not depend on feedback or prediction errors. However, analogously to BG-based learning, the learned knowledge increasingly depends on cortex, via the formation of cortico-cortical connections through systems consolidation. This systems consolidation leads to generalization, in that the cortico-cortical connections represent features common to the individual associations learned in the MTL. Sleep promotes this consolidation (though forgetting still occurs), apparently more reliably than for consolidation in BG-based learning (King et al. 2017). Longer-term retention seems to be worse for declarative than procedural memory (Foerde et al 2006). Declarative memory has traditionally been linked to the acquisition of events (episodic memory) and facts (semantic memory), though it is actually much more flexible: it appears to be able to learn many types of information, including both arbitrary information (e.g., items and idiosyncratic associations) and regularities, and both implicit and explicit knowledge. Note that declarative memory seems to be the only learning and memory system to underlie explicit knowledge, whereas it is one of many (including procedural memory) to subserve implicit knowledge (Ullman, 2016). Learning can be very rapid in the MTL, occurring even after one exposure ('one-shot learning'), though repeated exposures strengthen the memory traces. Learned information can be retrieved by recollection or 
by familiarity. MTL learning is flexible not only in that it can learn most information, but also (in contrast to automatized BG-based knowledge) in that this information can be used flexibly for new purposes. The MTL (especially the hippocampus) may be involved in prediction, possibly by using the current context to predict items likely to occur in that context (Henson \& Gagnepain 2010). MTL-based learning is closely linked to working memory, which plays important roles both in encoding and recall, including possibly maintaining different elements (e.g., those occurring at different times) to be associated (Ullman 2016). Not surprisingly, brain structures underlying working memory and related functions (e.g., recall and executive functions), such as portions of the BG and frontal cortex that underlie these functions (various parts of the striatum, as well as ventrolateral and other prefrontal regions) also play (related) roles in declarative memory (Scimeca \& Badre 2012, Van der Linden et al 2000).

Various material-, learning-, and subject-related factors modulate the relative dependence of learning and/or processing in declarative vs. procedural memory. Here we summarize these factors, though note that not all factors have been investigated for all types of knowledge learned in the BG (e.g., perceptuo-motor sequences, perceptual sequences, categories). Note also that various aspects of BG/MTL modulation have been referred to as redundancy, competition, and compensation; we discuss all of these here (Poldrack \& Packard 2003; Ullman 2015, 2016).

First, material-level factors can affect whether information is learned in declarative or procedural memory. Individual items (e.g., specific objects) - to the extent that an item can exist without associations - can be learned in the MTL, in particular perirhinal cortex (Davachi 2006), but might not be supported by procedural memory. Associations, in contrast, can be learned by either circuit. However, it appears that unique associations (e.g., Clemi's dog just smelled that rotten squirrel) can only be learned in the MTL, in particular the hippocampus, consistent with the role for the hippocampus in episodic memory (Davachi 2006). The BG can also learn associations, but apparently only under particular circumstances: gradually, with many presentations, in contexts involving prediction (e.g., given a squirrel, will Clemi's dog smell it?), with rapid feedback. Importantly, the regularities shared by the many presented associations will be learned, while any unique information in each association is lost. Thus, BGbased learning critically involves learning regularities (Hélie et al 2015). Nevertheless, regularities (commonalities) across associations can also emerge from MTL-based learning, which indeed underlies the formation of long-term semantic knowledge from multiple individual episodes containing that knowledge (e.g., learning that the capital of Burkina Faso is Ouagadougou from many episodes of learning 
that information). It remains unclear whether there is any kind of information or learning context that requires the BG for learning (i.e., that the MTL or other structures cannot learn), though it may be that learning (automatized) associations involving motor responses (whether in the input or output element of a learned pair) (Rose et al 2011) or trial-and-error learning (Hélie et al 2015) might necessitate the BG. Thus, it appears that MTL- and BG-based learning are somewhat but not completely redundant, in that both can learn associations, though not necessarily of the same sort in the same learning conditions.

Second, various learning-related factors modulate the relative reliance on the two circuits, for material that could be learned in either. Providing explicit information (e.g., of a sequence) can push learning towards the MTL (Schendan et al 2003). Similarly, encouraging attention to underlying patterns (e.g., telling participants there is an underlying category) can result in explicit awareness of these patterns, and working memory/executive function-dependent hypothesis-driven learning in the MTL (Ashby \& Maddox 2011). In contrast, BG-based learning is promoted in implicit versions of the same tasks (i.e., without providing explicit information or encouraging attention) - even more so when learning under dual-task conditions, which reduce attentional resources for searching for underlying patterns (Ashby \& Maddox 2011, Hazeltine et al 1997). Additionally, during learning, the MTL is often involved in and relied on during early stages (consistent with its more rapid learning abilities), whereas the BG appear to be involved throughout acquisition, and are increasingly relied on over the course of practice (though BG lesions can revert dependence to knowledge learned in the MTL) (Packard 2008, Schendan et al 2003). Thus, MTL-based representations can apparently inhibit (block) BG-based representations, and vice versa, depending on which is predominant. The two systems can therefore be thought to be in competition. Additionally, during later stages of learning, after more attention has been paid to the task and stimuli, explicit awareness of underlying patterns can emerge, potentially (again) increasing reliance on declarative memory (Hazeltine et al 1997). Finally, as mentioned above, whereas rapid feedback in tasks involving prediction seems to result in BG-based learning (even if initially learning might also involve the MTL), slow feedback or no feedback seem to lead primarily to MTL-based learning.

Third, within- and between-subject level factors can moderate the relative dependence on declarative vs. procedural memory. Any subject-level factor that enhances or depresses learning or processing in one system relative to the other may shift reliance towards the more functionally available system. One such within-subject factor is development. Whereas during infancy, MTL learning, as well as working memory and executive functions, do not appear to be well-developed, these improve during 
childhood, and may plateau during young adulthood (Lavenex \& Lavenex 2013, Welsh et al 1991). In contrast, BG learning may be relatively well-established early in childhood, but attenuate around adolescence (Janacsek et al 2012). Indeed, although sequence learning relies mainly on procedural memory in both children and adults, it appears to depend more on procedural memory in children than adults, but more on declarative memory in adults than children (Thomas et al 2004). Between subjects, MTL and/or BG learning can be modulated by various factors, such as genotype (e.g., different alleles of the gene for BDNF differentially affect MTL learning, while those for DARPP-32 modulate BG learning) and sex (females show MTL learning advantages as compared to males, likely due in part to estrogen) (Ullman 2016). This leads to predictions for the relative dependence of MTL vs. BG learning for these factors, though we are not aware of any studies clearly testing such predictions for (non-linguistic) sequence or category learning. More extreme cases of between-subject differences in MTL or BG functioning can be found in individuals with disorders. For example, amnesic patients with MTL damage likely rely on the BG for sequence learning (though it takes them longer than healthy controls, consistent with early MTL learning in controls) (Curran 1997). In contrast, patients with BG dysfunction, such as those with Parkinson's disease, appear to compensate with declarative memory for category and sequence learning (Ullman \& Pullman 2015). Finally, there seems to be a 'seesaw effect' such that BG dysfunction may lead to enhanced MTL functioning, and vice versa, perhaps due to the absence of inhibition from the other system (Ullman 2004).

\section{PROCEDURAL MEMORY IN LANGUAGE}

\section{Grammar}

Across different levels of language, linguistic elements are distributed sequentially and hierarchically into larger structures, following predictable patterns. Phonotactic rules constrain the combination of phonological segments (phonemes) into syllables, while grammatical rules constrain the combination of simple words and bound morphemes into complex words (e.g., meow + -ed; morphology) as well as phrases and sentences (e.g., 'the cat meowed'; syntax). The distributional patterns of linguistic elements can be captured by (various kinds of) structural representations of language, including syntactic trees generated by phrase-structure rules (e.g., Chomsky 1995; Pollard \& Sag 1994) and constructions in 
Construction Grammar (Goldberg 1995). These representations refer to abstract categories (e.g., Noun, Tense) in addition to individual words. They capture both local (adjacent) dependencies between successive elements (e.g., between Determiner and Noun, or 'the' and 'cat') and long-distance dependencies, which can be arbitrarily far apart. Within the hierarchically structured sequence, the probability of particular subsequent (downstream) elements ranges widely, from very low (e.g., noun phrase following noun phrase, as in 'The boy the girl kissed...') to very high and even deterministic (e.g., -ed following the verb stem for regular past-tense).

Grammar appears to rely heavily on procedural memory. First, grammar learning shares key characteristics with procedural memory. Both involve learning sequential and categorical knowledge, which is largely implicit in both cases. In both, long-distance dependencies are learned more slowly than local dependencies (see above, and Guasti 2017). Like learning in procedural memory, grammar is learned gradually and becomes largely automatic, that is, rapid, inflexible (grammatical relations become more rigidly delineated), and with decreased variability in performance (Love et al. 2009). Because linguistic input is generally fast-paced, successive elements (categories, words) can provide rapid feedback for internally generated predictions.

Direct evidence also links grammar learning to procedural memory. A meta-analysis of correlational studies found that individuals with better procedural learning abilities also show better learning or knowledge of grammar (but not of words) (Hamrick et al. 2018). This pattern was observed in children in their first language as well as adults learning a second language - though only at later stages of second language learning, when a greater dependence on knowledge learned in procedural memory is expected (at earlier stages second language correlated with learning abilities in declarative memory). The correlation between grammar and procedural learning held across different languages and linguistic structures (including syntax and inflectional morphology), and for both sequence and category learning tasks that depend on procedural memory. Grammar learning has also been tied specifically to the BG. A functional imaging neuroanatomical meta-analysis of early phases of adult language learning revealed that grammar (but not lexical) learning was associated with activation in the anterior caudate/putamen (and not the cerebellum) (Tagarelli et al. 2019), which we have seen underlies early phases of learning sequences and categories in procedural memory. Moreover, grammar learning that is predicted to rely particularly on procedural memory (e.g., with implicit/uninstructed training) showed anterior caudate/putamen involvement, whereas grammar learning predicted to rely especially on declarative 
memory (e.g., with explicit/instructed training) showed hippocampal involvement. Thus, consistent with MTL/BG learning of sequences and categories, evidence from correlational and neuroanatomical metaanalyses suggests that a) early stages of grammar learning involve both systems, but rely on declarative memory especially under conditions favoring learning in that system; b) during later stages, when grammatical knowledge in procedural memory has become predominant, grammar depends mainly on the procedural system. Finally, evidence from the lesion method also suggests that grammar learning relies on procedural memory. For example, early stage patients with Huntington's disease (characterized by striatal degeneration), are impaired at grammar learning (but relatively unimpaired at word learning) (De Diego-Balaguer et al. 2008).

Evidence suggests that procedural memory also underlies grammatical processing. In expressive language, the meaning of a message is transformed into a hierarchically structured sequence of words, morphemes, and syllables. In receptive language, linear sequences of syllables, morphemes, and words are translated into structured grammatical representations from which meaning is recovered. Here we focus on receptive grammatical processing, which is not only automatized (rapid, inflexible, and less variable; see above) in native language, but also involves prediction: in first (more than second) language, the automatized comprehension of rapidly unfolding sentences seems to depend importantly on the prediction of upcoming information, including word category or form (Kaan 2014). Given that automatization is linked to an increased reliance on cortico-cortical connections formed through BG-based learning, automatized grammatical processing should depend largely on cortical structures. Indeed, while grammar learning depends importantly on the BG (see above), grammatical processing in native speakers depends instead largely on cortex, as shown by converging evidence from neuroimaging, lesion (e.g., in patients with Broca's aphasia) and other methods (Ullman 2004, 2016). Indeed, a neuroanatomical metaanalysis of syntactic processing in native speakers implicated cortical regions but not the BG (or the cerebellum) (Walenski et al. 2019). Frontal regions, in particular premotor regions and Broca's region (inferior frontal gyrus), may play especially important roles (Ullman 2004, 2016; Walenski et al. 2019).

Just as sequences and categories learned in procedural memory can also be learned in declarative memory, evidence suggests that aspects of grammar can be learned in either system. However, the computational basis of the learning and representation of grammar seems to differ between the two systems. Procedural memory appears to subserve the learning of grammatical knowledge that underlies (real-time) combination (Ullman 2004, 2016), perhaps through the acquisition of (probabilistic) relations 
between categories or specific units that allow for the real-time prediction of downstream elements. In contrast, declarative memory can memorize (structured) chunks, such as 'meowed', 'the cat', or even 'NP VP', such as in Construction Grammar. Declarative memory can also learn explicit (or possibly implicit) rules (e.g., "nouns come after determiners") and appears to support generalization across similar stored associations (e.g., 'walk-walked' and 'talk-talked' may generalize to 'balk-balked') (Ullman 2004, 2016).

Similar factors appear to modulate the degree to which grammar and non-linguistic sequences/categories rely on each of the two systems. At least for adult language learning, both procedural and declarative memory support grammar during early stages, though in different ways: explicit knowledge, chunks, and similarity-based grammatical knowledge rely particularly on declarative memory, whereas implicit knowledge of grammatical regularities relies especially on procedural memory (Tagarelli et. al 2019). In contrast, at later stages grammar depends only on procedural memory (Hamrick et al. 2018). Local dependencies are particularly likely to be chunked, especially for highly frequent combinations; even surface syntactic structures may be stored (Ullman, 2016). Girls and women seem to depend more on declarative memory (e.g., by chunking and associative generalization) than boys and men for aspects of grammar, particularly for local dependencies (Ullman, 2016). This sex difference may be modulated by estrogen; higher levels of estrogen are associated with a greater reliance on chunked forms (Ullman, 2016). In disorders affecting procedural memory structures (e.g., Parkinson's disease, Broca's

aphasia), there appears to be a compensatory reliance on chunking of local dependencies, which may moreover interact with sex (with females relying more on chunking) (Ullman \& Pullman 2015; Johari et al. 2019).

\section{Lexicon}

Language also depends on the lexicon, which contains (at least) the arbitrary information that cannot be generated by the grammar, and thus must be stored. This minimally includes word forms (e.g., 'cat'), their meanings, and form-meaning associations, as well as information about irregular morphological forms (the form 'dug', and its link to 'dig'), the number and form of a verb's complements (e.g., 'devour' takes a single direct object), and larger arbitrary form-meaning mappings (e.g., idioms).

Although the declarative/procedural model has primarily linked the lexicon to declarative memory, some aspects of lexical learning and knowledge appear to rely on procedural memory. First, learning the 
phoneme sequences of word forms seems to depend partly on gradually and implicitly segmenting these sequences out of the speech stream (Singh et al. 2012). Such 'word segmentation' may depend on the anterior caudate/putamen during early phases of learning, underscoring its reliance on procedural memory (Karuza et al. 2013). Second, words whose meanings involve knowledge of motor skills that were likely learned in procedural memory (e.g., words for tools or actions) are also linked to the BG (Walenski et al. 2007). Third, closed-class words and morphemes (e.g., auxiliaries, inflectional affixes such as -ed), which are not tightly bound to conceptual meanings but depend strongly on grammatical structure, are more closely linked to the BG than open-class words (Ullman 2004, 2016). Despite the apparent suitability for procedural memory to learn aspects of conceptual/semantic categories (e.g., allowing one to group individual cat exemplars into a semantic category for cat) or form-meaning associations, we are not aware of any evidence along these lines.

The bulk of evidence ties lexical learning and knowledge to declarative memory (Ullman, 2004, 2016). First, correlational evidence shows that children with better declarative (but not procedural) learning abilities also show better lexical abilities (Hamrick et al. 2018). Second, consolidation patterns of newly learned word forms and semantic features are consistent with systems consolidation following hippocampal learning (Schreiner \& Rasch 2017). Third, amnesics with MTL lesions show word learning impairments (Davis \& Gaskell 2009). Fourth, neuroimaging studies have linked word learning to the hippocampus (Davis \& Gaskell 2009). Moreover, a neuroanatomical meta-analysis of adult language learning ties word learning to ventral stream structures, which are closely linked to the MTL (Tagarelli et. al, 2019). Finally, the N400 event-related potential component, which is found in response to various lexical/semantic manipulations, relies importantly on MTL structures, especially perirhinal cortex (Fernández \& Tendolkar 2006).

\section{Speech-sound representations}

Speech-sound representations are distributions of acoustic-phonetic features that can function as linguistic categories. They include contrastive (phonemic) and non-contrastive (phonetic) categories, as well as talker-specific information. The characteristics of speech-sound learning in infancy resonate with BG-based category learning. Young children acquire speech-sound categories implicitly via the gradual exposure to distributions of phonetic features. Whereas infants are perceptually flexible, they soon become less sensitive to features that are non-contrastive in their native language (Zhang et al. 2005). 
Speech-sound category learning has generally been investigated in adults. This research suggests that such learning relies initially on a 'reflective' learning system but during later stages on a 'reflexive' learning system, which have been respectively tied to declarative and procedural memory structures (Yi et al. 2014). Converging evidence suggests that automatization and other optimal outcomes of speechsound category learning are contingent on this switch from declarative to procedural memory (Yi et al. 2014). Factors that modulate the relative dependence of non-linguistic categories on the two systems play analogous roles in speech-sound category learning. Explicit knowledge of the speech-sound category label or the criteria of category membership increases reliance on declarative memory (Chandrasekaran et al. 2016). In contrast, learning relies more on procedural memory when rapid feedback is provided (Tricomi et al. 2006), which moreover dramatically improves the rate of category learning (Chandrasekaran et al. 2014). In sum, while both memory systems appear to be engaged in speech-sound category learning, efficient and ultimately successful learning of speech-sound categories seems to depend on the extent of procedural memory involvement.

This work in adults leads to the prediction that native-language speech-sound category learning in infants relies even more on procedural memory. Unlike in many adult studies, infants rarely encounter speech-sounds in isolation, but as elements of a sequence wherein successive elements provide rapid feedback. Moreover, the early-life weaknesses of declarative memory relative to procedural memory suggests that infants rely particularly on the latter. Taken together, procedural memory likely plays a predominant role in native speech-sound category learning.

\section{Articulation and speech production}

Articulatory sequences seem to be learned in procedural memory much like other perceptuo-motor skills. Learning to produce articulatory sequences appears to occur largely implicitly, and evidence suggests a role for rapid feedback in babbling behavior (Goldstein \& Schwade 2008), a precursor to articulation. Infants learn to articulate in the context of a rapidly changing vocal tract, necessitating relative flexibility in the motor program, whereas the motor program stabilizes once the articulatory apparatus has matured. Over the lifespan, the production of articulatory sequences (i.e., speech production) becomes automatized, in that variability is reduced and production rate increases (Smith \& Zelaznik 2004). 
Direct evidence from non-infant populations also ties articulatory learning to the BG. Studies of patients with Parkinson's disease implicate the BG in adapting pre-existing speech-motor sequences to auditory feedback, which by its nature occurs rapidly (Defne Abur et al. 2018). Furthermore, learning in the $\mathrm{BG}$ as a mechanism for adaptive vocal learning has been widely explored in songbirds, a model that shows clear behavioral and neural parallels with human vocal learning, an aspect of learning to articulate (Doupe \& Kuhl 1999). For example, FoxP2 expression in an avian correlate of the BG (area X) is positively associated with imitative vocal learning in songbirds (Haesler et al. 2007).

Speech production also relies on procedural memory circuitry (as well as the cerebellum). First, the BG are implicated in the timing of articulatory gestures, including in predicting that articulatory gestures will lead to the appropriately timed auditory and tactile feedback (Kotz \& Shwartze 2010). To illustrate, temporal processing impairments in patients with Parkinson's disease appear to be linked to deficits in speech production, which improve when external timing cues are provided (Thaut et al. 2001). Second, the BG act in concert with frontal regions, both in selecting the speech-motor programs apparently stored in the vicinity of BA 44, and in the initiation of these programs by supplementary motor area (SMA; Tourville \& Guenther, 2011). Indeed, patients with cortical lesions to BA 44/6 often present with acquired apraxia of speech, a disorder of speech-motor programming (Duffy 2006). Thus, procedural circuitry underlies not only the learning of speech-motor programs, but also the timing, prediction, selection, and initiation of these programs during speech production.

\section{Speech perception}

Speech perception (the process of mapping the auditory speech signal to meaning) involves constraining various sources of information, including cross-modal (visual-auditory-motor) information about the signal, various types of linguistic knowledge, and perceptual experience (McClelland et al. 2006). To accomplish this at the rapid rate of spoken language, evidence suggests that speech perception relies on predicting the upcoming signal based on current and prior information (Kutas et al. 2011). As such, language knowledge, including procedural memory-based knowledge (e.g. grammar, speech-sound categories, articulatory skills) will impact speech perception (Holt \& Lotto 2010). Furthermore, converging evidence links such prediction to BG-based temporal processing (Kotz \& Shwartze 2010). Thus, both language knowledge and temporally-sensitive prediction that depend on procedural memory are critical for efficient speech perception. 


\section{THE PDH: PRINCIPLES AND PREDICTIONS}

The PDH of any given disorder (e.g., DLD, dyslexia) posits that abnormalities of procedural memory brain structures, i.e., of cortico-BG-thalamo-cortical circuitry, can partly if not largely explain the disorder (Ullman 2004; Ullman \& Pierpont 2005). That is, such abnormalities can account for-and predict additional — core as well as secondary characteristics of the disorder in a substantial number of afflicted individuals. Such individuals may be termed as having Developmental Procedural circuit Disorder, or DPD. Thus, we do not claim that the PDH can explain all symptoms in all individuals diagnosed with any such disorder. Nevertheless, we suggest that it has substantial explanatory power for a number of language (and other) disorders, though the extent of this power likely varies across disorders. Given the large number of possible predictions that follow from our understanding of procedural memory as well its role in language, only key predictions are discussed here and examined below under each disorder.

The location(s), severity, and extent of the neuroanatomical abnormalities may vary across disorders, as well as across individuals within a disorder, in several ways (Ullman \& Pierpont 2005). Different gray and white matter 'levels' of the cortico-BG-thalamo-cortical circuitry can be affected, including within the BG itself. Moreover, abnormalities could involve different (sub)circuits (portions of the parallel circuitry), at any level (e.g., striatum, thalamus, cortex). In principle the direct or indirect pathways could be preferentially affected. Together, such anatomical variability could lead to substantial phenotypic variability within and between disorders, including in the type and severity of the deficits. For example, striatal dysfunction should lead to learning deficits while cortical dysfunction may primarily affect the processing of automatized skills. And anterior caudate/putamen abnormalities should mainly affect earlier phases of learning, while posterior striatal impairments affect later phases, including the process of automatization.

The etiology and pathology of the neuroanatomical abnormalities may also vary across and within disorders, since various genetic and environmental factors can cause the dysfunction of gray or white matter structures/pathways within procedural memory circuitry (Ullman \& Pierpont 2005). Therefore, like the acquired aphasias, disorders accounted for by the PDH may be best explained by their pattern of 
neuroanatomical abnormalities, largely independent of etiology or pathology (though different etiologies and pathologies could also lead to functional variability). The striatum may be especially vulnerable to dysfunction (Mitchell et al. 1999), perhaps (like the hippocampus) due in part to its relatively high level of learning-related plasticity, which is associated with high neuronal energy demands, and thus susceptibility (Bartsch \& Wulff 2015; Harris et al. 2012; Kreitzer \& Malenka 2008; Mitchell et al. 1999). Therefore abnormalities of the striatum may be particularly common. Nevertheless, any given etiology may affect brain structures more broadly, both within and in addition to procedural memory circuitry. Thus, other brain structures (e.g., cortex, the cerebellum) may also be affected in disorders explained by the PDH, if not directly by the underlying etiology, then indirectly through diaschesis or downstream functional effects. For example, striatal abnormalities impairing learning could affect frontal or temporal cortical processing of skills that were not normally learned.

Individuals with disorders explained by the PDH are not unable to learn. Their learning abilities may derive in part from procedural memory, since procedural memory dysfunction does not imply afunctionality, and thus some learning may take place within procedural memory itself. Additionally, the PDH posits that declarative memory remains relatively intact in individuals with DPD (Ullman \& Pierpont 2005; Ullman \& Pullman, 2015). Given the flexibility of declarative memory, it should support a vast range of information and capabilities in individuals with DPD, including by compensating for procedural memory and other dysfunctions. This in turn leads to further predictions. For example, impairments of functions that declarative memory can more easily compensate for (e.g., grammar more than motor functions, and within grammar local- more than long-distance dependencies, particularly higher frequency local relations) should be less evident and more likely to improve over time. Moreover, children with better declarative memory abilities should compensate more successfully, and may even compensate their way out of diagnosis. Individuals or groups with better declarative memory may therefore show a lower rate of diagnosis of these disorders (e.g., females vs. males; those with higher estrogen; val vs. met carriers of the val66met polymorphism of the BDNF gene) (Ullman \& Pullman 2015).

These patterns have various consequences (Ullman and Pierpont, 2005). First, all the expected sources of variability described above may contribute (though not exhaustively) to the heterogeneity across individuals within a disorder. Second, and in contrast, the commonalities across individuals of a disorder (e.g., grammatical difficulties in DLD), may be explained by shared dysfunction of procedural memory circuitry, even if the severity and etiology varies. Third, the expected variability, in particular 
differences in the location(s) of the anatomical abnormalities, should help explain differences between disorders. For example, motor-speech disorders likely result from dysfunction of motor circuits, while DLD or dyslexia may (also) involve other circuits. Dysfunction of additional structures (whether through direct or indirect effects) may also contribute to differences between disorders. Thus, disorders may profitably be distinguished, at least in part, on the basis of such expected anatomical differences. Finally, the commonalities and comorbidities among disorders (e.g., articulation and other motor problems found in both in DLD and motor-speech disorders) can be at least partly explained by overlapping abnormalities of procedural memory circuitry.

\section{DEVELOPMENTAL DISORDERS}

Various developmental disorders may be at least partly explained by the PDH. Here we focus on three (sets of) language-related disorders: DLD, developmental motor-speech disorders, and dyslexia. All are good candidates for the PDH on the basis of their core and additional symptoms, which involve deficits of functions that depend on cortico-BG-thalamo-cortical circuits (also see Krishnan et al. 2016). Indeed, as we will see, despite their differences, these disorders share multiple brain and behavioral characteristics linked to procedural memory circuitry. Moreover, within at least certain disorders the PDH unifies various impairments that have previously been targeted by different accounts (e.g., grammatical, working memory, phonological, and temporal processing deficits in DLD).

\section{Developmental language disorder (DLD)}

Consistent with the PDH, structural anomalies in DLD are found reliably (that is, a high proportion of studies show anomalies) in the BG, particularly the caudate nucleus, and in frontal cortex (Ullman \& Pierpont 2005, see Table; Mayes et al. 2015). Similarly, in functional imaging studies both frontal cortex (especially Broca's region) and the BG show reliable anomalies. The head of the caudate appears to be particularly implicated in DLD. No other brain structures seem to show as reliable structural or functional abnormalities, including superior temporal cortex, the cerebellum, and the MTL. Moreover, as expected by the PDH, various genetic and environmental factors associated with the BG (and in some cases with dopaminergic processes) seem to contribute to symptoms of DLD, including the FOXP2, CNTNAP2, 
SEMA6D, ANKK1, and D2D2 genes (Ercan-Sencicek et al. 2012; Newbury et al. 2010; Eicher et al. 2013), prenatal nicotine exposure (Eicher et al. 2013), and thiamine deficiency (Fattal et al. 2011).

Procedural memory has been shown to be impaired in DLD (indeed, after the PDH was proposed by Ullman \& Pierpont in 2005). First, meta-analysis has demonstrated deficits in the implicit SRT task in DLD (Lum et al. 2014; Clark \& Lum 2017). Impairments in DLD have also been found in grapho-motor learning (Adi-Japha et al. 2011), rotary pursuit (Lee \& Tomblin 2015; but see Hsu \& Bishop 2014), probabilistic category learning with rapid feedback (Kemeny \& Lukacs 2009; Lee \& Tomblin 2012; Lee \& Tomblin 2015; but see Mayor-Dubois et al. 2014), and perceptual sequence 'statistical learning' tasks such as word segmentation (Evans et al., 2009; Mayor-Dubois et al. 2014) - all tasks that depend on procedural memory. Thus, (early stage) learning of perceptuo-motor sequences, perceptual sequences, and categories is affected, specifically consistent with anterior caudate abnormalities (see section on procedural memory). The absence of learning deficits in some studies may be explained at least in part by insufficient power or by declarative memory compensation. (Note that implicit learning tasks that do not depend on procedural memory circuits are not predicted to be impaired; Conway et al. 2019). Some studies of procedural memory in DLD have found impairments primarily or only after post-training delays (Hedenius et al., 2011; Adi-Japha et al. 2011), also implicating consolidation deficits. Indeed, motor skills are impaired in DLD, in particular those involving sequences, across oral (speech and non-speech) and non-oral (e.g., hand) movements (Hill 2001; Ullman \& Pierpont 2005). Moreover, consistent with procedural memory impairments, temporal processing is impaired in DLD (e.g., in rhythm and meter perception, and of acoustic stimuli of short duration or presented in rapid succession) (Przybylski et al. 2013; Leonard 2014). Finally, other functions that depend on the BG, whether or not they are directly linked to procedural memory, are also impaired in DLD, including working memory and executive functions such as inhibition (Ullman \& Pullman 2005; Henry et al. 2012).

Grammar is clearly impaired in DLD (Leonard 2014; Ullman \& Pierpont 2005). Deficits are commonly found across syntax, morphology, and phonology, both in expressive and receptive language. Evidence links the grammar problems to procedural memory in the form of correlations between measures of grammar and procedural learning, including for both perceptuo-motor sequence and category learning (Hedenius et al. 2011; Mayor-Dubois et al. 2014). Particular problems seem to be found in those aspects of grammar that a) rely on procedural memory (especially if they are difficult to learn in this system), and b) cannot easily be compensated for by declarative memory (Ullman \& Pierpont 2005). Long-distance 
dependencies appear to be more problematic than local dependencies (Purdy et al. 2014), the latter of which are easier to learn in procedural memory (Guasti 2017) and easier to chunk in declarative memory (Ullman \& Pullman 2015). Previously encountered local dependencies that are higher frequency (e.g., 'walked') are less impaired than lower frequency ones, also suggesting chunking (Ullman \& Pierpont 2005). Similarly, canonical syntactic structures are relatively spared in DLD (Leonard \& Kueser 2019), consistent with learning structured chunks of abstract categories in declarative memory (e.g., in constructions). Aspects of grammar that likely depend importantly on declarative memory in typicallydeveloping individuals should also remain relatively intact in DLD. Indeed, this is the case with argument structure (Ullman \& Pierpont 2005). Grammar learning (e.g., in artificial grammar paradigms) is also impaired in DLD, especially for long(er)-distance dependencies that cannot easily be chunked (Hsu \& Bishop 2010). Moreover, automatization of grammar is problematic in DLD (Hsu \& Bishop 2010). Other procedural memory-related functions that are impaired in DLD may influence the grammatical problems. For example, syntactic processing in DLD seems linked to the temporal processing (timing) deficits in the disorder (Przybylski et al. 2013). In contrast, even though working memory can plays roles in grammar and is impaired in DLD, it does not appear to contribute to the grammatical problems (Lum et al. 2012).

Learning in declarative memory remains largely intact in DLD, especially for non-verbal material, but even in the verbal domain after controlling for working memory and language impairments (Lum et al 2012; Ullman \& Pullman 2015). Additionally, some evidence suggests that children with DLD might consolidate information in declarative memory better than typically-developing children (Lukacs et al. 2017). Consistent with intact declarative memory, various lines of evidence suggest that declarative memory compensates for the grammatical deficits, e.g., by chunking (see above), learning explicit rules, and associative generalization (Ullman and Pullman 2015). In one study grammatical abilities correlated with procedural learning in typically-developing children but declarative learning in children with DLD, suggesting DLD compensation by declarative memory (Lum et al. 2012). Some evidence suggests that whereas typical individuals show early left anterior negativities (fast, automatic, and linked to procedural memory) in response to syntactic impairments, those with DLD show N400s, which are linked to declarative memory (Ullman \& Pullman 2015). Moreover, the prevalence of DLD is higher in males than females, consistent with female advantages at declarative memory, and thus a particularly high level of declarative memory compensation by girls (Ullman \& Pullman 2015). 
Word learning and knowledge remain largely normal in DLD, unless they involve functions that depend on procedural memory and its neural substrates. Thus, children with DLD can learn new words (and in some cases show enhanced performance), especially in rich semantic contexts or with repetitive input, and appear to have normal lexical/semantic organization (Ullman \& Pierpont 2005). They also show largely intact N400s, both for content words and lexical/semantic processing (Ullman \& Pierpont 2005; Ullman \& Pullman 2015). In contrast, word learning that depends on procedural memory functions, such as word segmentation and learning in grammatical contexts, seems to be impaired in DLD (Evans et al., 2009; Mayor-Dubois et al. 2014; Ullman \& Pierpont 2005). This may help explain why children with DLD have smaller vocabularies than their age-matched peers (Lee 2011). Additionally, they appear to have shallower semantic representations for known words (McGregor 2009), consistent with procedural memory-based category learning difficulties, and have problems inhibiting competing words in word recognition (McMurray et al. 2014), consistent with deficits in executive function. Word finding and production are especially impaired in DLD (particularly with little contextual support and during rapid naming), as compared to receptive lexical abilities, consistent with abnormalities of frontal/BG circuits (which underlie recall) and the speech-motor (articulatory) difficulties in DLD (Ullman \& Pierpont 2005). Finally, children with DLD have more problems with function (closed-class) words than content (openclass words), and with verbs than nouns, consistent with the strong grammatical roles associated with function (vs. content) words and verbs (vs. nouns), and the action knowledge linked to verbs (Ullman \& Pierpont 2005).

How about other language-related functions that rely on procedural memory, such as speech-sound category learning, articulation, and speech perception? Although we are not aware of any DLD research on speech-sound category learning particularly linked to procedural memory, articulation may be affected in DLD (Hill 2001; Ullman \& Pierpont 2005). Moreover, speech perception (e.g., identifying words in noise) is impaired in the disorder (Ziegler et al. 2005). Although this impairment has generally been ascribed to various processing deficits (Leonard 2014; Ullman \& Pierpont 2005), it can also be explained by processes or knowledge linked to procedural memory that are critical for speech perception and are impaired in the disorder, namely speech-motor representations (Katz et al. 1992), perception of timing (Corriveau \& Goswami 2009), and prediction (Hestvik et al. 2010).

\section{Developmental motor-speech disorders}


Motor-speech disorders refer, as an umbrella term, to disorders in the production of speech. Idiopathic developmental motor-speech disorders include articulation disorder, childhood apraxia of speech (CAS, or verbal dyspraxia), and developmental stuttering. Articulation disorder refers to problems producing discrete sounds or classes of sounds, as well as sequences of sounds. In rare cases, children with severe articulation problems are diagnosed with CAS, which involves difficulties planning and programming motor movements in speech production in the absence of musculoskeletal atypicalities in the speech production apparatus (Bernthal et al. 2009). Thus, both articulation disorder and CAS describe problems with aspects of producing the motor sequences required for speech production. By contrast, developmental stuttering is a fluency disorder that affects the timing, patterning (e.g., prosody), and rhythm of speech (Alm 2004).

Neuroanatomical evidence suggests that procedural memory circuits are involved in developmental motor-speech disorders. Most such research has focused on affected members of the KE family, who can be characterized as having CAS (verbal dyspraxia) (Alcock et al 2000a). These individuals have a deleterious mutation of FOXP2, a gene strongly associated with the striatum, especially the caudate nucleus (but not the hippocampus) (Ullman \& Pierpont 2005). Broca's region and motor regions show the most consistent abnormalities across both structural and functional imaging in affected members, while additional structural anomalies are clustered in the caudate nucleus along with superior temporal cortex and the cerebellum (Ullman \& Pierpont, 2005, see Table). Although the neuroanatomical basis of other populations of CAS or articulation disorder has not been well studied, we are aware of one functional imaging study of nonword repetition in adolescents with articulation disorder, which found activation anomalies in the putamen, cerebellum, and various cortical regions, including the inferior frontal gyrus and SMA (Tkach et al. 2011). In individuals with developmental stuttering, a robust literature points to structural abnormalities in cortico-BG-thalamo-cortical circuits, such as reduced gray matter volumes in the putamen and some cortical regions, and altered connectivity among the putamen, thalamus, SMA, and other cortical regions (Alm 2004; Lu et al. 2010). Moreover, caudate head activity correlates with the severity of disfluencies in affected individuals (Giraud et al. 2008). Overall, the data suggest that the fluency symptoms of developmental stuttering may be rooted in the striatum and its associated circuitry (Alm 2004).

There is some, although not extensive, evidence linking these motor-speech conditions to procedural memory dysfunction. Children with articulation disorder have difficulties adapting their 
articulatory motor programs to perturbed auditory feedback, suggesting problems in BG-based articulatory learning (Terband et al. 2014). Adults with developmental stuttering are impaired at both perceptuo-motor and perceptual sequence learning (Smits-Bandstra \& De Nil 2007). Moreover, similar to DLD, all three conditions are associated with concomitant deficits in motor functions (Hill 2001; Busan et al. 2013), auditory timing (e.g., rhythm perception) (Alcock et al. 2000b; Kenney et al. 2006; Wieland et al. 2015), and working memory (Kenney et al. 2006; Bajaj 2007), all of which are tied to procedural memory or its neural substrates.

The most compelling behavioral evidence regarding the PDH for motor-speech disorders may be in their core speech-motoric and associated linguistic deficits. It would follow from the predicted role of BG-based learning in articulation that procedural memory deficits should impede the learning and automatization of speech-motoric sequences, which may lead to increased gestural variability between productions and slower speech rate. Indeed, both children with articulation disorder and CAS show both such symptoms (Flipsen Jr. 2002; Shriberg et al. 2003; Terband et al. 2011). Beyond these core characteristics, articulation disorder and CAS are both associated with deficits in phonology, morphology, and syntax (Alcock et al., 2000a; Mortimer \& Rvachew, 2010) and with eventual problems in learning how to read (Gillon \& Moriarty, 2007; Peterson et al. 2009). This suggests the possibility that these motorspeech disorders are associated with procedural impairments in these domains as well, consistent with their comorbidities with DLD and dyslexia. The core deficits of stuttering are also linked to procedural memory and its neural substrates. Indeed, their deficits in the timing and initiation of speech-motor programs for the fluent production of speech are expected from a dysfunction in the BG-SMA circuit (Alm, 2004). Moreover, developmental stuttering is associated with morphological and syntactic problems, and particular difficulties with verbs (Smith \& Weber 2017; Bauman et al. 2012), suggesting similarities in their procedural language impairments with DLD.

We are unaware of any studies that have investigated declarative memory directly in the three disorders, though it is predicted to remain largely intact. Nevertheless, some evidence suggests compensatory roles for this system. Declarative memory may help compensate for impairments in CAS, given that explicit strategies are effective behavioral therapies for this disorder (Dale \& Hayden 2013). Additionally, like DLD, syntactic anomalies yield N400s in developmental stuttering (Smith \& Weber 2017). Intriguingly, all three disorders are more prevalent in boys than girls (Bernthal et al. 2009; Yairi \& Ambrose 1999), and developmental stuttering is more likely to resolve by adulthood in women than in 
men (Porfert \& Rosenfield 1978), consistent with a female advantage at compensating with declarative memory.

\section{Developmental dyslexia}

Developmental dyslexia refers to reading disability involving problems in fluent decoding (processing the letter-sound, or grapheme-phoneme, mappings) that may cascade into reading comprehension problems - as opposed to reading comprehension deficits that may be secondary to language comprehension problems (Gough \& Tunmer, 1986; APA 2013). Structural anatomical abnormalities in dyslexia are found most consistently in superior temporal/temporoparietal and inferior/ventral temporal regions, as well as the cerebellum (Eckert et al. 2016), though they are also observed in other regions, including the putamen (Eckert et al., 2005; Pernet et al. 2009) and caudate head (Brown et al. 2001). Although functional imaging abnormalities must be interpreted carefully due to their task dependence, they have been observed in meta-analyses in the anterior caudate, putamen/globus pallidus, motor and inferior frontal regions, and the temporal regions listed above (Paulesu et al. 2014; Richlan et al 2011). In an SRT study of dyslexia, activation abnormalities were found in the putamen, as well as the SMA, cerebellum, and parietal regions (Menghini et al. 2006). Thus, procedural memory brain structures are implicated in dyslexia, including the basal ganglia (anterior caudate and putamen) and frontal (motor and inferior frontal) structures. It remains unclear whether the underlying etiologies affect the procedural circuit and/or other structures directly, or whether some of their abnormalities are (also) due to indirect effects (diaschesis, downstream effects).

As with DLD, procedural memory is impaired in dyslexia. Meta-analysis has demonstrated perceptuo-motor sequence learning deficits in the implicit SRT task (Lum et al. 2013; Clark \& Lum 2017). Impairments have also been found in perceptual sequence 'statistical' learning (Gabay et al. 2015a) and non-linguistic visual (weather prediction) and auditory category learning (Gabay et al. 2015b; Gabay \& Holt 2015). Thus, similar to DLD, perceptuo-motor sequence, perceptual sequence, and category learning are all impaired, consistent with anterior caudate abnormalities (but not with cerebellar anomalies; see procedural memory section). In addition, dyslexia is linked to deficits of other functions associated with

procedural memory and its underlying circuits, including impaired automaticity in speech production (Catts, 1989), (visual) artificial grammar learning difficulties (van Witteloostuijn et al. 2017), atypical (morpho-)syntactic development and processing (Scarborough 1991; Cantiani et al. 2013), atypical 
temporal processing (Farmer \& Klein 1995), impaired linguistic prediction (Huettig \& Brouwer, 2015), and poor working memory (Smith-Spark \& Fisk, 2007) and inhibition (Wang et al. 2012).

The reading problems in dyslexia may be at least partly explained by problems with procedural memory and its circuitry. First, the core decoding impairment in dyslexia is commonly attributed to the phonological deficits that clearly accompany the disorder, and often specifically to apparent problems with speech-sound representations (Ramus et al. 2003). These speech-sound representation problems have in turn been explained by atypical perception, such as impairments of rapid auditory processing or temporal processing (Farmer \& Klein 1995; Ramus et al. 2003). The PDH can account for at least some of these perceptual difficulties. Second, the PDH might expect impairments in learning speech-sound categories. Such impairments could result in a lack of cohesion in phonemic category representations, which indeed is consistent with observed weaknesses in dyslexia in phonemic anchoring (Ahissar 2007) and talker-specific adaptation (Perrachione et al. 2011), and with an over-reliance on non-contrastive features of speech (Serniclaes, 2011). Moreover, these characteristics appear to be consistent with learning (suboptimal) 'episodic' (instance specific) features of speech in declarative memory, rather than (optimal) categories in procedural memory (Shohamy et al. 2008). Third, magnocellular circuitry is closely linked with the dorsal stream (Goswami 2015), which in turn is closely linked to procedural memory (see above), suggesting that posited magnocellular deficits (see introduction) may be related to the impairments posited by the PDH. Finally, given the role of procedural memory in learning predictive associations, it may underlie learning grapheme-phoneme (and phoneme-grapheme) mappings (though these mappings are likely also learned in declarative memory, especially at early stages). Thus, procedural dysfunction could also contribute directly to impairments learning such mappings in dyslexia.

Declarative memory seems to remain largely intact in dyslexia. Non-verbal learning, including in implicit tasks such as spatial contextual learning, does not appear to be impaired (Ullman \& Pullman 2015). Verbal learning (including form-meaning mapping, as in word learning) is problematic, though the observed deficits may be explained by procedural memory-related dysfunctions, such as with phonology or working memory (Litt \& Nation 2014; Ullman \& Pullman 2015). Indeed, though encoding of verbal material (which depends on such functions) is impaired, retention of learned material seems to remain intact. For example, children with dyslexia may learn fewer words during encoding in a list-learning task but have no difficulty subsequently remembering the words they learned (Kramer et al. 2000). Some evidence even suggests that declarative memory may be enhanced in dyslexia (Ullman \& Pullman 2015). 
Finally, evidence suggests that declarative memory plays compensatory roles in dyslexia. Individuals with dyslexia appear to rely disproportionately on declarative memory for reading, using at least three strategies: chunking, a reliance on semantics, and the use of explicit knowledge (Ullman \& Pullman 2015). First, whereas people with dyslexia have particular problems reading made-up words, they are less impaired at reading real words (whose grapheme and/or phonological strings could have been memorized, i.e., chunked), especially those that occur that occur with higher frequency. Second, reading with supportive semantic contexts is particularly helpful in dyslexia, and improvements in reading during development appear to be due to an increased reliance on semantic knowledge. Third, explicit instruction of grapheme-phoneme correspondences ('phonics') is an effective therapy in dyslexia (Ehri et al. 2001). Indeed, successful behavioral interventions in dyslexia have been found to lead to increased (compensatory) activation in the hippocampus and other MTL structures, as well as increased hippocampal gray matter (Ullman \& Pullman 2015). As with DLD, syntactic violations have been found to lead to N400s in dyslexia (Cantiani et al. 2013), suggesting declarative memory compensation for syntactic impairments. Learning speech-sound categories seems to rely on declarative memory-based 'episodic' features in dyslexia (see just above) but relies mainly on procedural memory in typicallydeveloping individuals (see speech-sound representation section). Evidence also suggests that better declarative memory correlates with better reading in individuals with dyslexia but not in typicallydeveloping individuals (Ullman \& Pullman 2015). Finally, as with the other disorders, dyslexia may be more prevalent in boys than girls, though this pattern remains unclear (Hawke et al. 2009).

\section{Other developmental disorders}

The PDH may at least partially explain other disorders as well, whether or not they affect language functions. Indeed, abnormalities of procedural memory and its underlying circuitry, and compensation by declarative memory, have been implicated in a variety of other developmental disorders — which are often comorbid with each other and with the language disorders discussed above. These include ADHD, autism spectrum disorder, Tourette syndrome, obsessive-compulsive disorder, developmental coordination disorder, mathematical disability (e.g., developmental dyscalculia), and schizophrenia (Bradshaw \& Enticott, 2014; Clark \& Lum 2017; Evans \& Ullman 2016; Ullman, 2004; Ullman \& Pierpont 2005; Ullman \& Pullman 2015; Walenski et al., 2006, 2007, 2010). Interestingly, whereas in many of these disorders procedural memory seems to be impaired, in others (perhaps in subgroups) the abnormalities manifest as procedural memory enhancements, including of grammar (e.g., in Tourette syndrome and 
possibly autism spectrum disorder; Takács et al. 2018; Dye et al. 2016; Walenski et al., 2007, 2014). Finally, note that impairments of procedural memory and its neural substrates, as well as compensation by declarative memory, have been found in various adult-onset disorders, including Parkinson's disease and non-fluent/agrammatic aphasia (Ullman, 2004; Ullman \& Pullman, 2015). Thus, the core tenets of the PDH may extend beyond developmental disorders.

\section{CONCLUSION}

The PDH has numerous implications. In terms of basic research, the findings underscore the importance of the BG in language - in particular the anterior caudate nucleus, which likely plays a critical role in early phases of learning grammar and other aspects of language. Indeed, the evidence presented above not only strengthens the basic principles of the declarative/procedural model regarding lexicon and grammar (Ullman, 2004, 2015, 2016) but also extends them to other core aspects of language (speech sound representations, articulation, speech production, and speech perception), as well as other functions such as reading and math.

We emphasize that we have explored only a subset of the predictions that follow from the PDH. In particular, our quite specific understanding of procedural and declarative memory and their underlying circuitry, as well as of the language and non-language functions that depend on them, lead to a wide range of predictions and potentially promising lines of research - only some of which have begun to be explored. For example, disorders explained by the PDH might be expected to be associated with deficits of numerous functions and behaviors that may rely on the affected circuitry, as diverse as driving, social skills, habit formation, musical abilities, dorsal stream functions, BG-based selection, and even motivation and related affective functions. Our knowledge of declarative memory also leads to various intriguing possibilities. For example, are higher estrogen points of the menstrual cycle associated with fewer deficits? Do individuals with worse sleep show more impairments (since sleep supports declarative memory consolidation), and thus may sleep play a role in the disorders?

The PDH also makes translational predictions. It suggests the potential utility of pharmacological (e.g., dopaminergic), behavioral, and other interventions that have been successfully employed to enhance procedural memory, including in other disorders affecting its circuitry (de Vries et al. 2010; Ullman \& 
Pierpont 2005). Additionally, the compensatory role of declarative memory suggests the likely value of pharmacological and other interventions aimed at enhancing such compensation (Ullman \& Pullman 2015). Diagnoses of these disorders may be aided by the presence of particular neuroanatomical anomalies in procedural memory structures (and lack thereof elsewhere, such as in the MTL), as well as by the presence of deficits (e.g., long-distance grammatical dependencies) that are difficult to compensate for in declarative memory (Ullman \& Pullman 2015).

In sum, the PDH offers an explanatory framework for multiple aspects of the brain and behavioral correlates of various language (and other) developmental disorders, as well as their commonalities, comorbidities, and differences. It thus constitutes a powerful unifying neurocognitive account of these developmental disorders. Given the wide range of specific predictions that follow from the PDH, only the tip of the basic and translational research iceberg has been examined. We hope the present paper serves to guide research that probes the depths of this iceberg.

\section{REFERENCES}

Abla D, Katahira K, Okanoya K. 2008. On-line assessment of statistical learning by event-related potentials. J. Cogn. Neurosci. 20: 952-64.

Adi-Japha E, Strulovich-Schwartz O, Julius M (2011) Delayed motor skill acquisition in children with language impairment. Res Dev Disabil 32:2963-2971.

Ahissar, M. (2007). Dyslexia and the anchoring-deficit hypothesis. Trends in Cognitive Sciences, 11(11), 458-465.

Alcock, K. J., Passingham, R. E., Watkins, K. E., \& Vargha-Khadem, F. (2000a). Oral dyspraxia in inherited speech and language impairment and acquired dysphasia. Brain and Language, 75(1), 17-33.

Alcock, K. J., Passingham, R. E., Watkins, K., \& Vargha-Khadem, F. (2000b). Pitch and timing abilities in inherited speech and language impairment. Brain and Language, 75(1), 34-46. 
Alm, P. A. (2004). Stuttering and the basal ganglia circuits: a critical review of possible relations. Journal of Communication Disorders, 37(4), 325-369.

American Psychiatric Association (2013) Diagnostic and statistical manual of mental disorders: DMS-5 (American Psychiatric Association, Washington, D.C.) 5th Ed pp xliv, 947 p.

Ashby FG, Crossley MJ. 2012. Automaticity and multiple memory systems. Wiley Interdisciplinary Reviews: Cognitive Science 3: 363-76.

Ashby FG, Ennis JM, Spiering BJ. 2007. A neurobiological theory of automaticity in perceptual categorization. Psychol. Rev. 114: 632.

Ashby FG, Maddox WT. 2011. Human category learning 2.0. Ann. N. Y. Acad. Sci. 1224: 147-61.

Bajaj, A. (2007). Working memory involvement in stuttering: Exploring the evidence and research implications. Journal of Fluency Disorders, 32(3), 218-238.

Balleine BW, O'Doherty JP. 2010. Human and rodent homologies in action control: corticostriatal determinants of goal-directed and habitual action. Neuropsychopharmacology 35: 48.

Bartsch, T., \& Wulff, P. (2015). The hippocampus in aging and disease: from plasticity to vulnerability.

Bauman, J., Hall, N. E., Wagovich, S. A., Weber-Fox, C. M., \& Ratner, N. B. (2012). Past tense marking in the spontaneous speech of preschool children who do and do not stutter. Journal of Fluency Disorders, 37(4), 314-324.

Bernthal, J. E., Bankson, N. W., \& Flipsen, P. (2009). Articulation and phonological disorders: Speech sound disorders in children. Boston, MA: Pearson.

Bishop, D. V., Snowling, M. J., Thompson, P. A., Greenhalgh, T., and the CATALISE-2 Consortium (2017). Phase 2 of CATALISE: A multinational and multidisciplinary Delphi consensus study of problems with language development: Terminology. Journal of Child Psychology and Psychiatry, 58(10), 10681080 . 
Bostan, A. C., \& Strick, P. L. (2018). The basal ganglia and the cerebellum: nodes in an integrated network. Nature Reviews Neuroscience, 1.

Bradshaw, J. L., \& Enticott, P. G. (2014). Developmental disorders of the frontostriatal system: Neuropsychological, neuropsychiatric and evolutionary perspectives. Psychology Press.

Brown, W. E., Eliez, S., Menon, V., Rumsey, J. M., White, C. D., \& Reiss, A. L. (2001). Preliminary evidence of widespread morphological variations of the brain in dyslexia. Neurology, 56(6), 781-783.

Busan, P., D'Ausilio, A., Borelli, M., Monti, F., Pelamatti, G., Pizzolato, G., \& Fadiga, L. (2013). Motor excitability evaluation in developmental stuttering: a transcranial magnetic stimulation study. Cortex, 49(3), 781-792.

Catts, H. W. (1989). Speech production deficits in developmental dyslexia. Journal of Speech and Hearing Disorders, 54(3), 422-428.

Cantiani, C., Lorusso, M. L., Perego, P., Molteni, M., \& Guasti, M. T. (2013). Event-related potentials reveal anomalous morphosyntactic processing in developmental dyslexia. Applied Psycholinguistics, 34(6), 1135-1162.

Chandrasekaran, B., Yi, H. G., \& Maddox, W. T. (2014). Dual-learning systems during speech category learning. Psychonomic Bulletin \& Review, 21(2), 488-495.

Chandrasekaran, B., Yi, H. G., Smayda, K. E., \& Maddox, W. T. (2016). Effect of explicit dimensional instruction on speech category learning. Attention, Perception, \& Psychophysics, 78(2), 566-582.

Chomsky, Noam (1995). The Minimalist Program. MIT Press.

Clark, G. M., \& Lum, J. A. (2017). Procedural learning in Parkinson's disease, specific language impairment, dyslexia, schizophrenia, developmental coordination disorder, and autism spectrum disorders: A second-order meta-analysis. Brain and Cognition, 117, 41-48.

Conway, C.M., Arciuli, J., Lum, J.A.G., \& Ullman, M.T. (2019) . Seeing problems that may not exist: A reply to West et al.'s questioning of the procedural deficit hypothesis. Developmental Science. 
Corriveau, K. H., \& Goswami, U. (2009). Rhythmic motor entrainment in children with speech and language impairments: tapping to the beat. Cortex, 45(1), 119-130.

Curran T. 1997. Higher-order associative learning in amnesia: Evidence from the serial reaction time task. J. Cogn. Neurosci. 9: 522.

Dale, P. S., \& Hayden, D. A. (2013). Treating speech subsystems in childhood apraxia of speech with tactual input: The PROMPT approach. American Journal of Speech-Language Pathology.

Davachi L. 2006. Item, context and relational episodic encoding in humans. Curr. Opin. Neurobiol. 16: 693-700.

Davis, M. H., \& Gaskell, M. G. (2009). A complementary systems account of word learning: Neural and behavioural evidence. Philosophical Transactions of the Royal Society of London, 364(1536), 3773-3800.

De Diego-Balaguer, R., Couette, M., Dolbeau, G., Dürr, A., Youssov, K., \& Bachoud-Lévi, A. C. (2008). Striatal degeneration impairs language learning: evidence from Huntington's disease. Brain, 131(11), 2870-2881.

Defne Abur, R., Lester-Smith, R. A., Daliri, A., Lupiani, A. A., Guenther, F. H., \& Stepp, C. E. (2018). Sensorimotor adaptation of voice fundamental frequency in Parkinson's disease. PloS One, 13(1), e0191839.

Delgado MR, Miller MM, Inati S, Phelps EA. 2005. An fMRI study of reward-related probability learning. Neuroimage 24: 862-73.

de Vries MH, Ulte C, Zwitserlood P, Szymanski B, \& Knecht S (2010) Increasing dopamine levels in the brain improves feedback-based procedural learning in healthy participants: an artificial-grammar-learning experiment. Neuropsychologia 48(11):3193-3197.

Doupe, A. J., \& Kuhl, P. K. (1999). Birdsong and human speech: common themes and mechanisms. Annual review of neuroscience, 22(1), 567-631. 
Doyon J, Bellec P, Amsel R, Penhune V, Monchi O, et al. 2009. Contributions of the basal ganglia and functionally related brain structures to motor learning. Behavioral Brain Research 199: 61-75.

Draganski B, Kherif F, Klöppel S, Cook PA, Alexander DC, et al. 2008. Evidence for segregated and integrative connectivity patterns in the human basal ganglia. J. Neurosci. 28: 7143-52.

Duffy, J. R. (2006). Apraxia of speech in degenerative neurologic disease. Aphasiology, 20(6), 511-527.

Durrant SJ, Cairney SA, Lewis PA. 2012. Overnight consolidation aids the transfer of statistical knowledge from the medial temporal lobe to the striatum. Cereb. Cortex 23: 2467-78.

Dye, C.D., Walenski, M., Mostofsky, S. H., \& Ullman, M.T. (2016). A verbal strength in children with Tourette syndrome? Evidence from a non-word repetition task. Brain and Language. 160, pp. 61-70.

Eckert, M. A., Berninger, V. W., Vaden Jr, K. I., Gebregziabher, M., \& Tsu, L. (2016). Gray matter features of reading disability: a combined meta-analytic and direct analysis approach. eNeuro, 3(1).

Eckert, M. A., Leonard, C. M., Wilke, M., Eckert, M., Richards, T., Richards, A., \& Berninger, V. (2005). Anatomical signatures of dyslexia in children: unique information from manual and voxel based morphometry brain measures. Cortex, 41(3), 304-315.

Ehri, L. C., Nunes, S. R., Stahl, S. A., \& Willows, D. M. (2001). Systematic phonics instruction helps students learn to read: Evidence from the National Reading Panel's meta-analysis. Review of Educational Research, 71(3), 393-447.

Eichenbaum, H. (2012). Memory systems. Handbook of Psychology, Second Edition, 3.

Eicher JD, et al. (2013) Associations of Prenatal Nicotine Exposure and the Dopamine Related Genes ANKK1 and DRD2 to Verbal Language. PLoS ONE 8(5):e63762.

Elbro, C., Dalby, M., \& Maarbjerg, S. (2011). Language-learning impairments: a 30-year follow-up of language-impaired children with and without psychiatric, neurological and cognitive difficulties. International Journal of Language \& Communication Disorders, 46(4), 437-448. 
Ercan-Sencicek AG, et al. (2012) A balanced $\mathrm{t}(10 ; 15)$ translocation in a male patient with developmental language disorder. Eur. J. Med. Genet. 55(2):128-131.

Evans, T.M., \& Ullman, M.T. (2016). An extension of the procedural deficit hypothesis from developmental language disorders to mathematical disability. Frontiers in Psychology. 7, 1318.

Evans JL, Saffran JR, Robe-Torres K (2009) Statistical learning in children with specific language impairment. Journal of Speech, Language, and Hearing Research 52:321-355.

Farmer, M. E., \& Klein, R. M. (1995). The evidence for a temporal processing deficit linked to dyslexia: A review. Psychonomic Bulletin \& Review, 2(4), 460-493.

Fattal I, Friedmann N, \& Fattal-Valevski A (2011) The crucial role of thiamine in the development of syntax and lexical retrieval: A study of infantile thiamine deficiency. Brain 134(6):1720-1739.

Fernández, G. \& Tendolkar, I. (2006). The rhinal cortex: Gatekeeper of the declarative memory system. Trends in Cognitive Sciences, 10(8), 358-362.

Flipsen Jr, P. (2002). Longitudinal changes in articulation rate and phonetic phrase length in children with speech delay. Journal of Speech, Language, and Hearing Research.

Foerde, K. E., Knowlton, B. J., \& Poldrack, R. A. (2006). Retention of classification learning after training under single and dual task conditions. Paper presented at the Society for Neuroscience Abstracts, Atlanta, GA, USA.

Foerde K, Shohamy D. 2011a. Feedback timing modulates brain systems for learning in humans. $J$. Neurosci. 31: 13157-67.

Foerde K, Shohamy D. 2011b. The role of the basal ganglia in learning and memory: insight from Parkinson's disease. Neurobiol. Learn. Mem. 96: 624-36.

Frank MJ. 2005. Dynamic Dopamine Modulation in the Basal Ganglia: A Neurocomputational Account of Cognitive Deficits in Medicated and Non-medicated Parkinsonism. J. Cogn. Neurosci. 17: 51-72. 
Gabay, Y., \& Holt, L. L. (2015). Incidental learning of sound categories is impaired in developmental dyslexia. Cortex, 73, 131-143.

Gabay, Y., Thiessen, E. D., \& Holt, L. L. (2015a). Impaired statistical learning in developmental dyslexia. Journal of Speech, Language, and Hearing Research, 58(3), 934-945.

Gabay, Y., Vakil, E., Schiff, R., \& Holt, L. L. (2015b). Probabilistic category learning in developmental dyslexia: Evidence from feedback and paired-associate weather prediction tasks. Neuropsychology, 29(6), 844.

Gillon, G. T., \& Moriarty, B. C. (2007). Childhood apraxia of speech: Children at risk for persistent reading and spelling disorder. In Seminars in speech and language (Vol. 28, No. 01, pp. 048-057). Thieme Medical Publishers, New York, NY.

Giraud, A. L., Neumann, K., Bachoud-Levi, A. C., von Gudenberg, A. W., Euler, H. A., Lanfermann, H., \& Preibisch, C. (2008). Severity of dysfluency correlates with basal ganglia activity in persistent developmental stuttering. Brain and language, 104(2), 190-199.

Goldberg, Adele (1995). Constructions: A construction grammar approach to argument structure. Chicago/London: University of Chicago Press.

Goldstein, M. H., \& Schwade, J. A. (2008). Social feedback to infants' babbling facilitates rapid phonological learning. Psychological science, 19(5), 515-523.

Goswami, U. (2015). Sensory theories of developmental dyslexia: three challenges for research. Nature Reviews Neuroscience, 16(1), 43.

Gough, P. B., \& Tunmer, W. E. (1986). Decoding, reading, and reading disability. Remedial and special education, 7(1), 6-10.

Grahn JA, Rowe JB. 2009. Feeling the beat: premotor and striatal interactions in musicians and nonmusicians during beat perception. J. Neurosci. 29: 7540-48. 
Graybiel AM, Grafton ST. 2015. The striatum: where skills and habits meet. Cold Spring Harb. Perspect. Biol. 7: a021691.

Guasti, M. T. (2017). Language acquisition: The growth of grammar. MIT press.

Haesler, S., Rochefort, C., Georgi, B., Licznerski, P., Osten, P., \& Scharff, C. (2007). Incomplete and inaccurate vocal imitation after knockdown of FoxP2 in songbird basal ganglia nucleus Area X. PLoS biology, 5(12), e321.

Hamrick, P., Lum, J. A., \& Ullman, M. T. (2018). Child first language and adult second language are both tied to general-purpose learning systems. Proceedings of the National Academy of Sciences, 115(7), 14871492.

Hardwick, R. M., Rottschy, C., Miall, R. C., \& Eickhoff, S. B. (2013). A quantitative meta-analysis and review of motor learning in the human brain. Neuroimage, 67, 283-297.

Harris, J. J., Jolivet, R., \& Attwell, D. (2012). Synaptic energy use and supply. Neuron, 75(5), $762-777$.

Hawke, J. L., Olson, R. K., Willcut, E. G., Wadsworth, S. J., \& DeFries, J. C. (2009). Gender ratios for reading difficulties. Dyslexia, 15(3), 239-242.

Hazeltine E, Grafton ST, Ivry R. 1997. Attention and stimulus characteristics determine the locus of motor-sequence encoding. A PET study. Brain 120 ( Pt 1): 123-40.

Hedenius, M., Persson, J., Tremblay, A., Adi-Japha, E., Veríssimo, J., Dye, C.D., Alm, P., Jennische, M., Tomblin, J.B., and Ullman, M.T. (2011). Grammar predicts procedural learning and consolidation deficits in children with specific language impairment. Research in Developmental Disabilities. 32(6). 2362-2375.

Hélie S, Ell SW, Ashby FG. 2015. Learning robust cortico-cortical associations with the basal ganglia: An integrative review. Cortex 64: 123-35

Hélie S, Roeder JL, Ashby FG. 2010a. Evidence for cortical automaticity in rule-based categorization. $J$. Neurosci. 30: 14225-34 
Hélie S, Waldschmidt JG, Ashby FG. 2010b. Automaticity in rule-based and information-integration categorization. Attention, Perception, \& Psychophysics 72: 1013-31.

Henry, L. A., Messer, D. J., \& Nash, G. (2012). Executive functioning in children with specific language impairment. Journal of child psychology and psychiatry, 53(1), 37-45.

Henson RN, Gagnepain P. 2010. Predictive, interactive multiple memory systems. Hippocampus 20: 1315-26.

Hestvik, A., Schwartz, R. G., \& Tornyova, L. (2010). Relative clause gap-filling in children with specific language impairment. Journal of Psycholinguistic Research, 39(5), 443-456.

Hill, E. L. (2001). Non-specific nature of specific language impairment: a review of the literature with regard to concomitant motor impairments. International journal of language \& communication disorders, 36(2), 149-171.

Holt, L. L., \& Lotto, A. J. (2010). Speech perception as categorization. Attention, Perception, \& Psychophysics, 72(5), 1218-1227.

Hsu, H. J., \& Bishop, D. V. (2010). Grammatical difficulties in children with specific language impairment: Is learning deficient?. Human development, 53(5), 264-277.

Hsu, H.J. and Bishop, D.V. (2014) Sequence-specific procedural learning deficits in children with specific language impairment. Dev. Sci. 17, 352-365

Huettig, F., \& Brouwer, S. (2015). Delayed anticipatory spoken language processing in adults with dyslexia-evidence from eye-tracking. Dyslexia, 21(2), 97-122.

Janacsek K, Fiser J, Nemeth D. 2012. The best time to acquire new skills: age-related differences in implicit sequence learning across the human lifespan. Developmental Science 15: 496-505.

Janacsek K, \& Nemeth D. 2012. Predicting the future: From implicit learning to consolidation. Int. J. Psychophysiol. 83: 213-21. 
Johari, K., Walenski, M., Reifegerste, J., Ashrafi, F., \& Ullman, M.T. (2019, online). Sex, dopamine, and hypokinesia: A study of inflectional morphology in Parkinson's disease. Neuropsychology.

Kaan, E. (2014). Predictive sentence processing in L2 and L1: What is different? Linguistic approaches to Bilingualism, 4(2), 257-282.

Karuza, E. A., Newport, E. L., Aslin, R. N., Starling, S. J., Tivarus, M. E., \& Bavelier, D. (2013). The neural correlates of statistical learning in a word segmentation task: An fMRI study. Brain and Language, 127(1), 46-54.

Katz, W. F., Curtiss, S., \& Tallal, P. (1992). Rapid automatized naming and gesture by normal and language-impaired children. Brain and Language, 43, 623-623.

Kemeny, F., \& Lukacs, A. (2009). Impaired procedural learning in language impairment: Results from probabilistic categorization. Journal of Clinical and Experimental Neuropsychology, iFirst, 1-12.

Kenney, M. K., Barac-Cikoja, D., Finnegan, K., Jeffries, N., \& Ludlow, C. L. (2006). Speech perception and short-term memory deficits in persistent developmental speech disorder. Brain and Language, 96(2), 178-190.

King BR, Hoedlmoser K, Hirschauer F, Dolfen N, Albouy G. 2017. Sleeping on the motor engram: the multifaceted nature of sleep-related motor memory consolidation. Neurosci. Biobehav. Rev. 80: 1-22

Kotz, S. A., \& Schwartze, M. (2010). Cortical speech processing unplugged: a timely subcortico-cortical framework. Trends in Cognitive Sciences, 14(9), 392-399.

Kramer, J. H., Knee, K., \& Delis, D. C. (2000). Verbal memory impairments in dyslexia. Archives of Clinical Neuropsychology, 15(1), 83-93.

Kreitzer, A. C., \& Malenka, R. C. (2008). Striatal plasticity and basal ganglia circuit function. Neuron, 60(4), 543-554.

Krishnan, S., Watkins, K. E., \& Bishop, D. V. (2016). Neurobiological basis of language learning difficulties. Trends in Cognitive Sciences, 20(9), 701-714. 
Kutas, M., DeLong, K. A., \& Smith, N. J. (2011). A look around at what lies ahead: Prediction and predictability in language processing. Predictions in the brain: Using our past to generate a future, 190207.

Lavenex P, Lavenex PB. 2013. Building hippocampal circuits to learn and remember: insights into the development of human memory. Behav. Brain Res. 254: 8-21.

Lee, J. (2011). Size matters: Early vocabulary as a predictor of language and literacy competence. Applied Psycholinguistics, 32(1), 69-92.

Lee, J. C., \& Tomblin, J. B. (2012). Reinforcement learning in young adults with developmental language impairment. Brain and Language, 123(3), 154-163.

Lee, J. C., \& Tomblin, J. B. (2015). Procedural learning and individual differences in language. Language Learning and Development, 11(3), 215-236.

Leonard L, B. (2014 ) Children with specific language impairment (MIT Press )2nd Ed p 496.

Leonard, L. B., \& Kueser, J. B. (2019) Five overarching factors central to grammatical learning and treatment in children with developmental language disorder. International Journal of Language \& Communication Disorders.

Litt, R. A., \& Nation, K. (2014). The nature and specificity of paired associate learning deficits in children with dyslexia. Journal of Memory and Language, 71(1), 71-88.

Love, T., Walenski, M., \& Swinney, D. (2009). Slowed speech input has a differential impact on on-line and off-line processing in children's comprehension of pronouns. Journal of Psycholinguistic Research, 38(3), 285-304.

Lu, C., Peng, D., Chen, C., Ning, N., Ding, G., Li, K., Yang, Y. \& Lin, C. (2010). Altered effective connectivity and anomalous anatomy in the basal ganglia-thalamocortical circuit of stuttering speakers. Cortex, 46(1), 49-67.

Lukacs, A., Kemeny, F., Lum, J.A.G., \& Ullman, M.T. (2017). Learning and overnight retention in declarative memory in specific language impairment. PLOS ONE 12(1): e0169474. 
Lum, J.A.G., Conti-Ramsden, G.M., Morgan, A.T., \& Ullman, M.T. (2014). Procedural learning deficits in Specific Language Impairment (SLI): A meta-analysis of serial reaction time task performance. Cortex. $51,1-10$.

Lum, J. A., Conti-Ramsden, G., Page, D., \& Ullman, M. T. (2012). Working, declarative and procedural memory in specific language impairment. Cortex, 48(9), 1138-1154.

Lum, J. A., Ullman, M. T., \& Conti-Ramsden, G. (2013). Procedural learning is impaired in dyslexia: Evidence from a meta-analysis of serial reaction time studies. Research in Developmental Disabilities, 34(10), 3460-3476.

Mayes A, K., Reilly S, \& Morgan A, T. (2015) Neural correlates of childhood language disorder: A systematic review. Dev. Med. Child Neurol. 57(8):706-717.

Mayor-Dubois, C. et al. (2014) Nondeclarative learning in children with specific language impairment: predicting regularities in the visuomotor, phonological, and cognitive domains. Child Neuro-psychol. 20, $14-22$

McClelland, J. L., Mirman, D., \& Holt, L. L. (2006). Are there interactive processes in speech perception?. Trends in Cognitive Sciences, 10(8), 363-369.

McGregor, K. K. (2009). Semantic deficits across populations. Handbook of Child Language Disorders, 365.

McMurray, B., Munson, C. \& Tomblin, J.B. (2014) Individual differences in language ability are related to variation in word recognition, not speech perception: Evidence from eye-movements. Journal of Speech Language and Hearing Research, 57, 1344-1362.

Menghini, D., Hagberg, G. E., Caltagirone, C., Petrosini, L., \& Vicari, S. (2006). Implicit learning deficits in dyslexic adults: An fMRI study. NeuroImage, 33(4), 1218-1226.

Middleton FA, Strick PL. 1996. The temporal lobe is a target of output from the basal ganglia. Proceedings of the National Academy of Sciences USA 93: 8683-8687. 
Mitchell, I. J., Cooper, A. J., \& Griffiths, M. R. (1999). The selective vulnerability of striatopallidal neurons. Progress in Neurobiology, 59(6), 691-719.

Mortimer, J., \& Rvachew, S. (2010). A longitudinal investigation of morpho-syntax in children with speech sound disorders. Journal of Communication Disorders, 43(1), 61-76.

Newbury DF, Fisher SE, \& Monaco AP (2010) Recent advances in the genetics of language impairment. Genome Med. 2(6):1-8.

Nicolson, R. I., \& Fawcett, A. J. (2007). Procedural learning difficulties: reuniting the developmental disorders?. Trends in Neurosciences, 30(4), 135-141.

Packard MG. 2008. Neurobiology of procedural learning in animals In Concise learning and memory: The editor's selection, ed. JH Byrne, pp. 341-56. London: Elsevier Science \& Technology Books

Paulesu, E., Danelli, L., \& Berlingeri, M. (2014). Reading the dyslexic brain: multiple dysfunctional routes revealed by a new meta-analysis of PET and fMRI activation studies. Frontiers in Human Neuroscience, 8,830 .

Penhune VB, Steele CJ. 2012. Parallel contributions of cerebellar, striatal and M1 mechanisms to motor sequence learning. Behav. Brain Res. 226: 579-91.

Perrachione, T. K., Del Tufo, S. N., \& Gabrieli, J. D. (2011). Human voice recognition depends on language ability. Science, 333(6042), 595-595.

Pernet, C. R., Poline, J. B., Demonet, J. F., \& Rousselet, G. A. (2009). Brain classification reveals the right cerebellum as the best biomarker of dyslexia. BMC neuroscience, 10(1), 67.

Peterson, R. L., Pennington, B. F., Shriberg, L. D., \& Boada, R. (2009). What influences literacy outcome in children with speech sound disorder?. Journal of Speech, Language, and Hearing Research.

Poldrack RA, Clark J, Pare-Blagoev EJ, Shohamy D, Creso Moyano J, et al. 2001. Interactive memory systems in the human brain. Nature 414: 546-50. 
Poldrack RA, Packard MG. 2003. Competition among multiple memory systems: converging evidence from animal and human brain studies. Neuropsychologia 41: 245-51.

Pollard, Carl, and Sag, Ivan A. (1994). Head-Driven Phrase Structure Grammar. Chicago: University of Chicago Press.

Porfert, A. R., \& Rosenfield, D. B. (1978). Prevalence of stuttering. Journal of Neurology, Neurosurgery \& Psychiatry, 41(10), 954-956.

Przybylski, L., Bedoin, N., Krifi-Papoz, S., Herbillon, V., Roch, D., Léculier, L., Kotz, S.A., \& Tillmann, B. (2013). Rhythmic auditory stimulation influences syntactic processing in children with developmental language disorders. Neuropsychology, 27(1), 121.

Purdy, J. D., Leonard, L. B., Weber-Fox, C., \& Kaganovich, N. (2014). Decreased sensitivity to longdistance dependencies in children with a history of specific language impairment: Electrophysiological evidence. Journal of Speech, Language, and Hearing Research, 57(3), 1040-1059.

Ramus, F., Rosen, S., Dakin, S. C., Day, B. L., Castellote, J. M., White, S., \& Frith, U. (2003). Theories of developmental dyslexia: insights from a multiple case study of dyslexic adults. Brain, 126(4), 841-865.

Remillard, G. (2008). Implicit learning of second-, third-, and fourth-order adjacent and nonadjacent sequential dependencies. The Quarterly Journal of Experimental Psychology, 61(3), 400-424.

Richlan, F., Kronbichler, M., \& Wimmer, H. (2011). Meta-analyzing brain dysfunctions in dyslexic children and adults. Neuroimage, 56(3), 1735-1742.

Rose M, Haider H, Salari N, Büchel C. 2011. Functional dissociation of hippocampal mechanism during implicit learning based on the domain of associations. J. Neurosci. 31: 13739-45

Scarborough, H. S. (1991). Early syntactic development of dyslexic children. Annals of Dyslexia, 41(1), 207-220.

Schendan HE, Searl M, Melrose R, Stern C. 2003. An FMRI study of the role of the medial temporal lobe in implicit and explicit sequence learning. Neuron 37: 1013-25. 
Schreiner, T., \& Rasch, B. (2017). The beneficial role of memory reactivation for language learning during sleep: A review. Brain and language, 167, 94-105.

Scimeca JM, Badre D. 2012. Striatal contributions to declarative memory retrieval. Neuron 75: 380-92.

Seger CA, Miller EK. 2010. Category learning in the brain. Annu. Rev. Neurosci. 33: 203-19.

Seger CA, Peterson EJ, Cincotta CM, Lopez-Paniagua D, Anderson CW. 2010. Dissociating the contributions of independent corticostriatal systems to visual categorization learning through the use of reinforcement learning modeling and Granger causality modeling. Neuroimage 50: 644-56.

Serniclaes, W. (2011). Allophonic perception in dyslexia: An overview. Escritos de psicología, 4(2), 2534.

Shohamy, D., Myers, C. E., Kalanithi, J., \& Gluck, M. A. (2008). Basal ganglia and dopamine contributions to probabilistic category learning. Neuroscience \& Biobehavioral Reviews, 32(2), 219-236.

Shriberg, L. D., Green, J. R., Campbell, T. F., Mcsweeny, J. L., \& Scheer, A. R. (2003). A diagnostic marker for childhood apraxia of speech: The coefficient of variation ratio. Clinical Linguistics \& Phonetics, 17(7), 575-595.

Singh, L., Steven Reznick, J., \& Xuehua, L. (2012). Infant word segmentation and childhood vocabulary development: a longitudinal analysis. Developmental Science, 15(4), 482-495.

Smith, A., \& Weber, C. (2017). How stuttering develops: The multifactorial dynamic pathways theory. Journal of Speech, Language, and Hearing Research, 60(9), 2483-2505.

Smith, A., \& Zelaznik, H. N. (2004). Development of functional synergies for speech motor coordination in childhood and adolescence. Developmental Psychobiology, 45(1), 22-33.

Smith-Spark, J. H., \& Fisk, J. E. (2007). Working memory functioning in developmental dyslexia. Memory, 15(1), 34-56.

Smits-Bandstra, S., \& De Nil, L.F. (2007). Sequence skill learning in persons who stutter: implications for cortico-striato-thalamo-cortical dysfunction. Journal of Fluency Disorders, 32(4), 251-278. 
Tagarelli, K.M., Shattuck, K.F., Turkeltaub, P.E., \& Ullman, M.T. (2019) Language learning in the adult brain: A neuroanatomical meta-analysis of lexical and grammatical learning. NeuroImage, 193, 178-200.

Takács, Á., Kóbor, A., Chezan, J., Éltető, N., Tárnok, Z., Nemeth, D., Ullman, M.T., \& Janacsek, K. (2018). Is procedural memory enhanced in Tourette syndrome? Evidence from a sequence learning task. Cortex. 100, 84-94.

Terband, H., Maassen, B., Van Lieshout, P. H. H. M., \& Nijland, L. (2011). Stability and composition of functional synergies for speech movements in children with developmental speech disorders. Journal of Communication Disorders, 44(1), 59-74.

Terband, H., Van Brenk, F., \& van Doornik-van der Zee, A. (2014). Auditory feedback perturbation in children with developmental speech sound disorders. Journal of Communication Disorders, 51, 64-77.

Thaut, M. H., McIntosh, K. W., McIntosh, G. C., \& Hoemberg, V. (2001). Auditory rhythmicity enhances movement and speech motor control in patients with Parkinson's disease. Functional Neurology, 16(2), 163-172.

Thomas KM, Hunt RH, Vizueta N, Sommer T, Durston S, et al. 2004. Evidence of Developmental Differences in Implicit Sequence Learning: An fMRI Study of Children and Adults. J. Cogn. Neurosci. 16: $1339-51$.

Tkach, J. A., Chen, X., Freebairn, L. A., Schmithorst, V. J., Holland, S. K., \& Lewis, B. A. (2011). Neural correlates of phonological processing in speech sound disorder: a functional magnetic resonance imaging study. Brain and Language, 119(1), 42-49.

Tourville, J. A., \& Guenther, F. H. (2011). The DIVA model: A neural theory of speech acquisition and production. Language and Cognitive Processes, 26(7), 952-981.

Tricomi, E., Delgado, M. R., McCandliss, B. D., McClelland, J. L., \& Fiez, J. A. (2006). Performance feedback drives caudate activation in a phonological learning task. Journal of Cognitive Neuroscience, 18(6), 1029-1043. 
Turk-Browne NB, Scholl BJ, Chun MM, Johnson MK. 2009. Neural evidence of statistical learning: Efficient detection of visual regularities without awareness. J. Cogn. Neurosci. 21: 1934-45.

Ullman MT, Pullman MY. 2015. A compensatory role for declarative memory in neurodevelopmental disorders. Neurosci. Biobehav. Rev. 51: 205-22.

Ullman MT. 2004. Contributions of memory circuits to language: The declarative/procedural model. Cognition 92: 231-70.

Ullman MT. 2015. The declarative/procedural model: A neurobiologically motivated theory of first and second language In Theories in second language acquisition, ed. B VanPatten, J Williams, pp. 135-58. New York: Routledge.

Ullman MT. 2016. The declarative/procedural model: a neurobiological model of language learning, knowledge, and use In Neurobiology of language, pp. 953-68: Elsevier.

Ullman, M. T., \& Pierpont, E. I. (2005). Specific language impairment is not specific to language: The procedural deficit hypothesis. Cortex, 41(3), 399-433.

Vakil E, Bloch A, Cohen H. 2017. Anticipation measures of sequence learning: manual versus oculomotor versions of the serial reaction time task. The Quarterly Journal of Experimental Psychology 70: 579-89.

Van der Linden M, Meulemans T, Marczewski P, Collette F. 2000. The relationships between episodic memory, working memory, and executive functions: The contribution of the prefrontal cortex. Psychol. Belg. 40: 275-97

van Witteloostuijn, M., Boersma, P., Wijnen, F., \& Rispens, J. (2017). Visual artificial grammar learning in dyslexia: A meta-analysis. Research in Developmental Disabilities, 70, 126-137.

Waldschmidt JG, Ashby FG. 2011. Cortical and striatal contributions to automaticity in informationintegration categorization. Neuroimage 56: 1791-802. 
Walenski, M., Europa, E., Caplan, D., \& Thompson, C. K. (2019, online). Neural networks for sentence comprehension and production: An ALE-based meta-analysis of neuroimaging studies. Human brain mapping.

Walenski, M., Mostofsky, S. H. \& Ullman, M. T. (2014). Inflectional morphology in high-functioning autism: Evidence for speeded grammatical processing. Research in Autism Spectrum Disorders. 8, 16071621.

Walenski, M., Mostofsky, S. H., \& Ullman, M. T. (2007). Speeded processing of grammar and tool knowledge in Tourette's syndrome. Neuropsychologia, 45. 2447-2460.

Walenski, M., Tager-Flusberg, H., and Ullman, M. T. (2006). Language in Autism. In S. O. Moldin and J. L. R. Rubenstein (Eds.), Understanding Autism: From Basic Neuroscience to Treatment. Boca Raton, FL: Taylor and Francis Books.

Walenski, M., Weickert, T.W., Maloof, C.J., Ullman, M.T. (2010). Grammatical processing in schizophrenia: Evidence from morphology. Neuropsychologia, 48. 262-269.

Welsh MC, Pennington BF, Groisser DB. 1991. A normative-developmental study of executive function: A window on prefrontal function in children. Dev. Neuropsychol. 7: 131-49.

Wieland, E. A., McAuley, J. D., Dilley, L. C., \& Chang, S. E. (2015). Evidence for a rhythm perception deficit in children who stutter. Brain and Language, 144, 26-34.

Wilkinson L, Tai YF, Lin CS, Lagnado DA, Brooks DJ, et al. 2014. Probabilistic classification learning with corrective feedback is associated with in vivo striatal dopamine release in the ventral striatum, while learning without feedback is not. Hum. Brain Mapp. 35: 5106-15.

Yairi, E., \& Ambrose, N. G. (1999). Early childhood stuttering I: Persistency and recovery rates. Journal of Speech, Language, and Hearing Research, 42(5), 1097-1112.

Yi, H. G., Maddox, W. T., Mumford, J. A., \& Chandrasekaran, B. (2014). The role of corticostriatal systems in speech category learning. Cerebral Cortex, 26(4), 1409-1420. 
Wang, L. C., Tasi, H. J., \& Yang, H. M. (2012). Cognitive inhibition in students with and without dyslexia and dyscalculia. Research in Developmental Disabilities, 33(5), 1453-1461.

Ziegler, J. C., Pech-Georgel, C., George, F., Alario, F. X., \& Lorenzi, C. (2005). Deficits in speech perception predict language learning impairment. Proceedings of the National Academy of Sciences, 102(39), 14110-14115.

Zhang, Y., Kuhl, P. K., Imada, T., Kotani, M., \& Tohkura, Y. I. (2005). Effects of language experience: neural commitment to language-specific auditory patterns. NeuroImage, 26(3), 703-720. 